International Journal of Bifurcation and Chaos, Vol. 29, No. 7 (2019) 1930017 16 pages)

(C) The Author(s)

DOI: $10.1142 /$ S0218127419300179

\title{
Nested Closed Invariant Curves in Piecewise Smooth Maps
}

\author{
Viktor Avrutin \\ Institute for Systems Theory and Automatic Control, \\ University of Stuttgart, Pfaffenwaldring 9, \\ 70550 Stuttgart, Germany \\ avrutin@ist.uni-stuttgart.de \\ Zhanybai T. Zhusubaliyev \\ Department of Computer Science, Southwest State University, \\ 50 Years of October Str. 94, 305040, Kursk, Russia \\ zhanybai@hotmail.com
}

Received December 20, 2018

\begin{abstract}
The paper describes how several coexisting stable closed invariant curves embedded into each other can arise in a two-dimensional piecewise-linear normal form map. Phenomena of this type have been recently reported for a piecewise smooth map, modeling the behavior of a power electronic DC-DC converter. In the present work, we demonstrate that this type of multistability exists in a more general class of models and show how it may result from the well-known period adding bifurcation structure due to its deformation so that the phase-locking regions start to overlap. We explain how this overlapping structure is related to the appearance of coexisting stable closed invariant curves nested into each other.

By means of detailed, numerically calculated phase portraits we hereafter present an example of this type of multistability. We also demonstrate that the basins of attraction of the nested stable invariant curves may be separated from each other not only by repelling closed invariant curves, as previously reported, but also by a chaotic saddle. It is suggested that the considered kind of multistability is a generic phenomenon in piecewise smooth dynamical systems.
\end{abstract}

Keywords: Piecewise smooth systems; two-dimensional piecewise-linear border collision normal form; multistability; closed invariant curves.

\section{Introduction}

Multistability is one of the common phenomena in the theory of nonlinear dynamical systems. Indeed, a coexistence of attractors has been observed in many fields of engineering and applied science such as power electronics Baneriee \& Verghese, 2001; Zhusubaliyev \& Mosekilde, 2003], mechanical engineering Feudel et al. 1996: BlażejczykOkolewska \& Kapitaniak, 1998|, neuroscience [Foss et al., 1996], chemistry Marmillot et al., 1991], optics Brambilla et al., 1991], and condensed matter physics [Prengel et al., 1994].

A distinguishing feature of multistable systems is their sensitivity to noise: an arbitrarily small

\footnotetext{
This is an Open Access article published by World Scientific Publishing Company. It is distributed under the terms of the Creative Commons Attribution 4.0 (CC-BY) License. Further distribution of this work is permitted, provided the original work is properly cited.
} 
level of noise may cause a sudden transition from one attractor to another, i.e. a fundamentally unpredictable behavior of the system [Feudel, 2008]. A broad discussion of the role of the multistability in complex dynamics can be found in a series of publications bv Feudel et al. Feudel. 2008: Pisarchik \& Feudel, 2014; Hens et al., 2015; Feudel et al., 2018]. For example, the global dynamics of a general class of multistable smooth systems with a large number of coexisting attractors and the influence of noise on the systems behavior is discussed in [Pisarchik \& Feudel, 2014; Hens et al., 2015; [Feudel et al., 2018].

Multistability in piecewise smooth systems is also a well-known phenomenon ZZhusubaliyev \& Mosekilde, 2003; Do \& Lai, 2008; Pavlovskaia et al., 2010; Liu \& Chávez, 2017]. The mechanisms leading to multistability in piecewise smooth systems are presently of interest for many researchers. In particular, using the well known piecewise-linear bordercollision normal form Nusse \& Yorke, 1992], it has been reported in [Do \& Lai, 2008] that the number of coexisting attracting cycles grows as the system approaches the Hamiltonian limit. Moreover, using the same normal form, Simpson 2014 has provided the conditions for the coexistence of an infinite number of attracting cycles in piecewise smooth maps. Border-collision bifurcations leading to the appearance of coexisting attractors (so-called multiple attractor bifurcations) are described in [Kapitaniak \& Maistrenko, 1998; Dutta et al., 1999; Avrutin et al., 2011.

One can distinguish between different kinds of multistability depending on the kinds of coexisting attractors. So far, mainly coexisting stable $m$-cycles, $m \geq 1$, have been considered in the literature. Recently however, Zhusubaliyev et al. Zhusubaliyev et al., 2011; Zhusubaliyev \& Mosekilde, 2015] showed that piecewise smooth systems can exhibit an unusual type of multistability. In the reported case, several stable closed invariant curves coexist, being nested one into each other. Hereby, their basins of attraction are separated by repelling closed invariant curves.

The reported case raises several questions. Is this specific kind of multistability sufficiently generic, so that it can be observed in the piecewiselinear border-collision normal form? Which cycles may form the attracting and repelling invariant curves nested into each other? Even more importantly, what is the mechanism leading to this kind of multistability?
The present paper is organized as follows. In Sec. 2. we recall the definition of the 2D bordercollision normal form. The period adding bifurcations structure in this map is discussed in Sec. 3. We show that this structure, appearing first in its classical form, may undergo a certain deformation, which leads to the onset of multistability. A particular example of the resulting structure in the phase space is reported in Sec. 4. In this example, the map shows five attracting closed invariant curves whose basins are separated from each other by two repelling closed invariant curves and two chaotic saddles. The structure of the phase space in this example is described in detail, focusing not only on the coexisting attractors but also on their basin boundaries. Section 5 concludes the paper.

\section{Piecewise-Linear Normal Form Map}

It is well known in the theory of nonsmooth systems, that many dynamical phenomena related to border-collisions can be examined by means of a piecewise-linear approximation to the Poincaré map in the neighborhood of the border-crossing fixed point, expressed as the piecewise-linear $2 \mathrm{D}$ normal form

$$
\begin{aligned}
X_{n+1} & =F\left(X_{n}, \mu\right), \\
F(X, \mu) & = \begin{cases}A_{\mathcal{L}} X+B \mu & \text { if } x \leq 0 ; \\
A_{\mathcal{R}} X+B \mu & \text { if } x>0\end{cases}
\end{aligned}
$$

with

$$
X=\left(\begin{array}{l}
x \\
y
\end{array}\right), \quad A_{\mathcal{L} / \mathcal{R}}=\left(\begin{array}{rr}
\tau_{\mathcal{L} / \mathcal{R}} & 1 \\
-\delta_{\mathcal{L} / \mathcal{R}} & 0
\end{array}\right), \quad B=\left(\begin{array}{l}
1 \\
0
\end{array}\right)
$$

originally introduced in [Nusse \& Yorke, 1992]. The phase plane of the map (11) is divided into two regions $\mathcal{L}=\{(x, y) \mid x \leq 0, y \in \mathbb{R}\}$ and $\mathcal{R}=$ $\{(x, y) \mid x>0, y \in \mathbb{R}\}$. Here the matrices $A_{\mathcal{L} / \mathcal{R}}$ are the Jacobians in the regions $\mathcal{L}$ and $\mathcal{R}$, and $\tau_{\mathcal{L} / \mathcal{R}}, \delta_{\mathcal{L} / \mathcal{R}}$ are their traces and determinants, respectively.

Let us choose the parameters such that $\delta_{\mathcal{L}}<1$ and $\delta_{\mathcal{R}}>1$. Then, the conditions

$$
\begin{aligned}
-\left(1+\delta_{\mathcal{L}}\right) & <\tau_{\mathcal{L}}<1+\delta_{\mathcal{L}}, \\
-2 \sqrt{\delta_{\mathcal{R}}} & <\tau_{\mathcal{R}}<2 \sqrt{\delta_{\mathcal{R}}}
\end{aligned}
$$

ensure that both for $\mu<0$ and $\mu>0$, map (1) has a fixed point, which is attracting for $\mu<0$ and a spiral repeller for $\mu>0$ [Zhusubalivev et al., 2006]. 
As the parameter $\mu$ is varied from negative to positive values, the fixed point of map (11) moves from $\mathcal{L}$ to $\mathcal{R}$ and undergoes a border-collision at $\mu=0$. If the parameters $\tau_{\mathcal{L}}$ and $\tau_{\mathcal{R}}$ satisfy the conditions (21), after this border-collision, one can observe a large variety of dynamical phenomena, including attracting closed invariant curves, associated with quasiperiodic or phase-locked dynamics Zhusubalivev et al., 2006. Moreover, as demonstrated below, this border-collision can result in the appearance of several coexisting stable closed invariant curves nested into each other.

\section{Deformation of Period Adding Structure}

In the present study we are interested in the mechanism leading to the appearance of several coexisting stable closed invariant curves, nested one into the other. To this end, we consider the bifurcations structure of map (11) in the parameter plane $\left(\tau_{\mathcal{L}}, \tau_{\mathcal{R}}\right)$ for $-0.2<\tau_{\mathcal{R}}<0.4$ and $-0.52<\tau_{\mathcal{L}}<0.022$ at the fixed values of the determinants $\delta_{\mathcal{L}}=0.5$, $\delta_{\mathcal{R}}=1.8094$.

In the following, stable and unstable $m$-cycles of map (11) are referred to as $\mathcal{O}_{m}^{s}$ and $\mathcal{O}_{m}^{u}$, respectively, and $\mathcal{P}_{m}, m \geq 1$, denotes the stability region of an $m$-cycle $\mathcal{O}_{m}^{s}$ in the $\left(\tau_{\mathcal{L}}, \tau_{\mathcal{R}}\right)$ parameter plane.

Moreover, to make the bifurcations structure easier to recognize we use the following nonlinear re-parametrization

$$
\tau_{\mathcal{L}}=h\left(\tau_{\mathcal{R}}\right)=a_{2} \tau_{\mathcal{R}}^{2}+a_{1} \tau_{\mathcal{R}}+a_{0}+\varepsilon
$$

where the coefficients $a_{0}=-0.127027, a_{1}=$ $-0.753981, a_{2}=-0.426519$ are obtained numerically by fitting the middle curve of the region $\mathcal{P}_{43}$. Proceeding in this way, we consider the parameter $\varepsilon$ instead of $\tau_{\mathcal{L}}$. It can be easily shown that in the considered ranges of the parameters $\tau_{\mathcal{L}}, \tau_{\mathcal{R}}$ the function $h$ is locally monotonous, and hence the transformation between parameter planes $\left(\tau_{\mathcal{L}}, \tau_{\mathcal{R}}\right)$ and $\left(\varepsilon, \tau_{\mathcal{R}}\right)$ is topology-preserving.

The bifurcation structure in the lower part of the region in the $\left(\varepsilon, \tau_{\mathcal{R}}\right)$ parameter plane presented in Fig. 1(a) is well recognizable. Indeed, we observe here a typical period adding structure, formed by sausage-like phase-locking regions of cycles with arbitrary high periods, as described by many authors (see, for instance, Yang \& Had, 1987; Campbell et al., 1996; Zhusubalivev et al., 2002; Zhusubalivev \& Mosekilde, 2008; Simpson \&
Meiss, 2009; Simpson, 2017, 2018]). A typical bifurcation diagram across this structure is shown in Fig. 2. One can easily recognize the usual shape of the period diagram reflecting the addition of the periods (so, for example, the region $\mathcal{P}_{133}$ is located between the regions $\mathcal{P}_{43}$ and $\mathcal{P}_{90}$, the region $\mathcal{P}_{223}$ is located between the regions $\mathcal{P}_{133}$ and $\mathcal{P}_{90}$, and so on) and the devil's-staircase-like structure of the associated rotation numbers.

In this structure, if the rotation number is rational, the dynamics of the map (11) is periodic, otherwise it is quasiperiodic (see Fig. 2), but in both cases there exists an attracting closed invariant curve. Wher the rotation number is rational, the closed invariant curve contains a pair of cycles of the same period, one of which is stable, while the other is a saddle. As an example, Fig. 廿(a) shows the phase portrait of the map at the parameter point inside the region $\mathcal{P}_{51}$ marked with $a$ in Fig. 1. The shown closed invariant curve $\mathcal{C}_{51}$ is formed by a saddlefocus connection between the 51-saddle $\mathcal{O}_{51}^{u}$ and the attracting 51-focus $\mathcal{O}_{51}^{s}$. Note that the considered paranneter value is below the border-collision fold bifurcation curve $\xi^{4}$ which represents a boundary of the region $\mathcal{P}_{4}$. On the boundary $\xi^{4}$ a pair of 4 -cycles appear, while below this boundary the closed invariant curve is the only attractor. When the parameters are varied across the border-collision fold bifurcation boundary of this part of the region $\mathcal{P}_{51}$, one observes the appearance of nonrobust stable closed invariant curve with quasiperiodic dynamics, as illustrated in Fig. 4(b).

With increasing values of $\tau_{\mathcal{R}}$, the period adding structure undergoes several transformations, as one can clearly see in the upper part of Fig. 1. Some of the involved cycles become unstable, so that the assoclated periodicity regions [as, for example, the regions $\mathcal{P}^{35}$ and $\mathcal{P}^{39}$ marked in Fig. [1(b)] cease to exist. Moreover, as $\tau_{\mathcal{R}}$ increases, the regions forming the period adding structure start to overlap which leads to the onset of multistability. This is clearly visible in Fig. 1(b): for example, for sufficiently large values of $\tau_{\mathcal{R}}$, the region $\mathcal{P}_{43}$ overlaps with some of the regions previously located betwe en the regions $\mathcal{P}_{39}$ and $\mathcal{P}_{43}$ with some of the regions previously located between $\mathcal{P}_{43}$ and $\mathcal{P}_{47}$. An example of the resulting bifurcation structure is shown in Fig. 3. As one can see, the structure does not resemble the period adding in any way, and is affected by a massive coexistence of several stable cycles. 


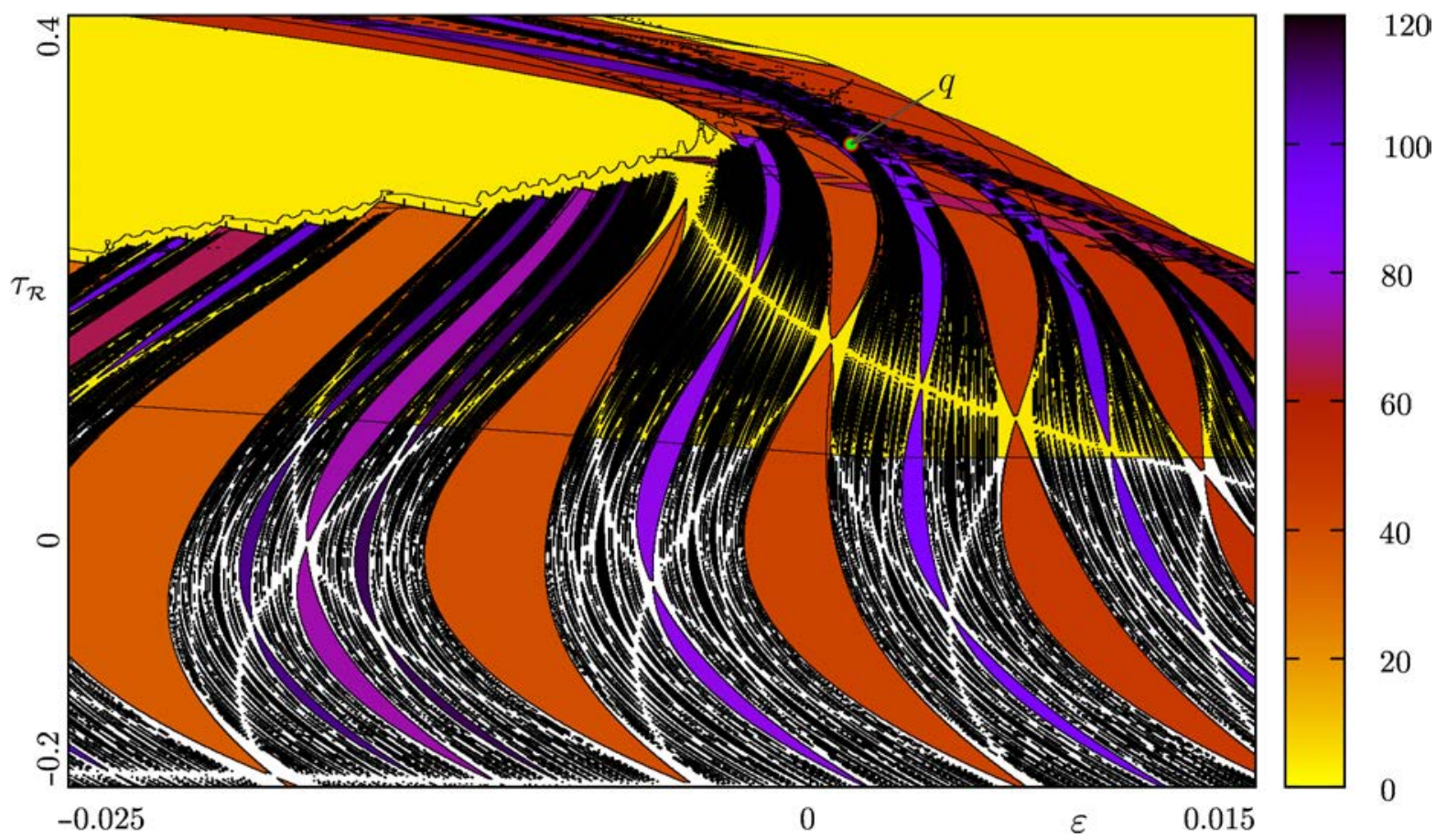

(a)

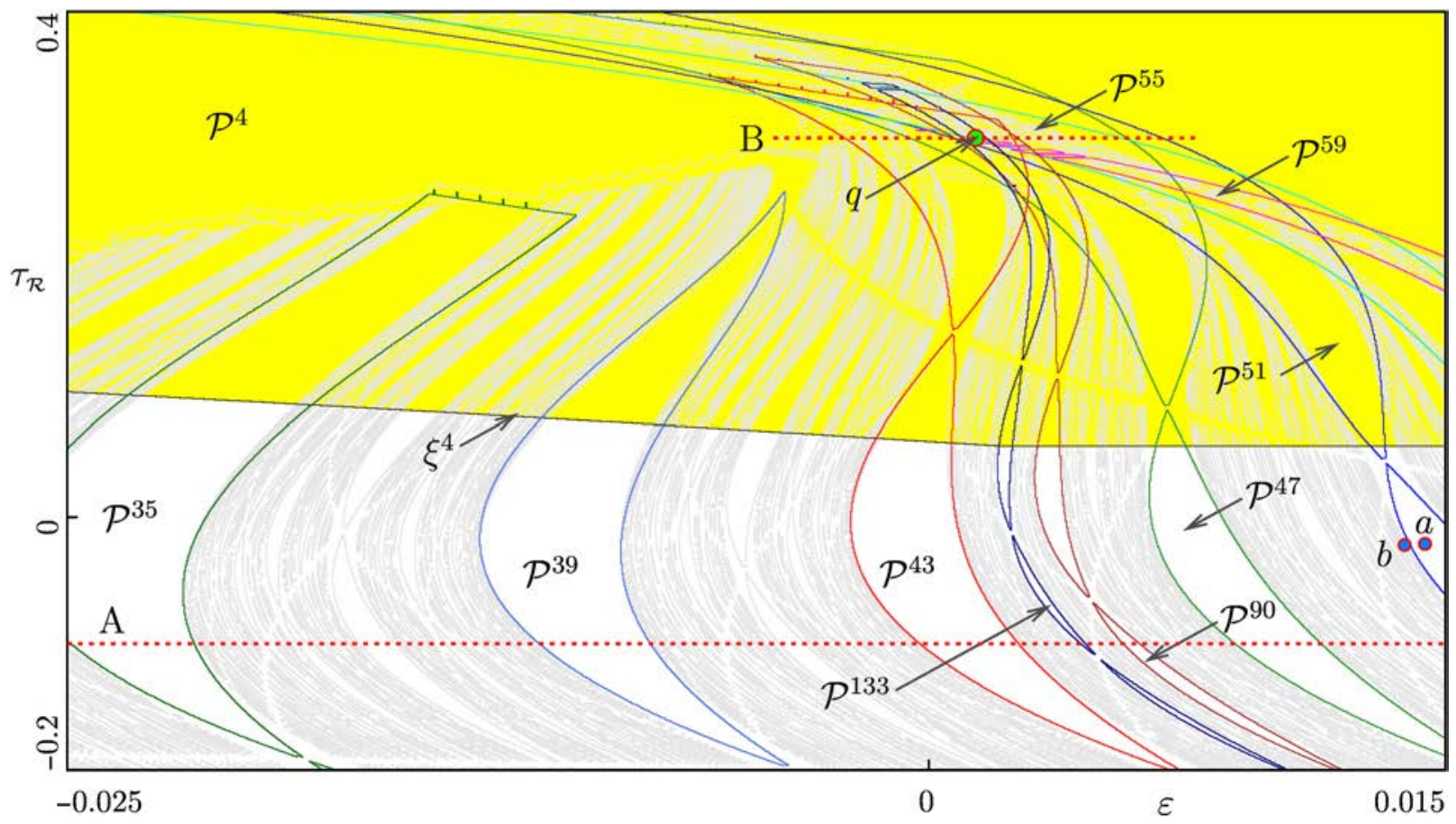

(b)

Fig. 1. (a) Overview of the bifurcation structure in the $\left(\varepsilon, \tau_{\mathcal{R}}\right)$ parameter plane leading to the appearance of several attracting closed invariant curves nested into each other. (b) $\mathcal{P}_{4}, \mathcal{P}_{43}, \mathcal{P}_{47}, \mathcal{P}_{51}, \mathcal{P}_{55}, \mathcal{P}_{59}, \mathcal{P}_{90}, \mathcal{P}_{133}$ denote regions of periodicity of the corresponding cycles overlapping at the point $q$. The parameter paths $\mathrm{A}$ and B indicated by dashed lines correspond to Figs. 2 and 3 respectively. The phase portraits at the points marked with $a$ and $b$ are shown in Fig. 4 . 


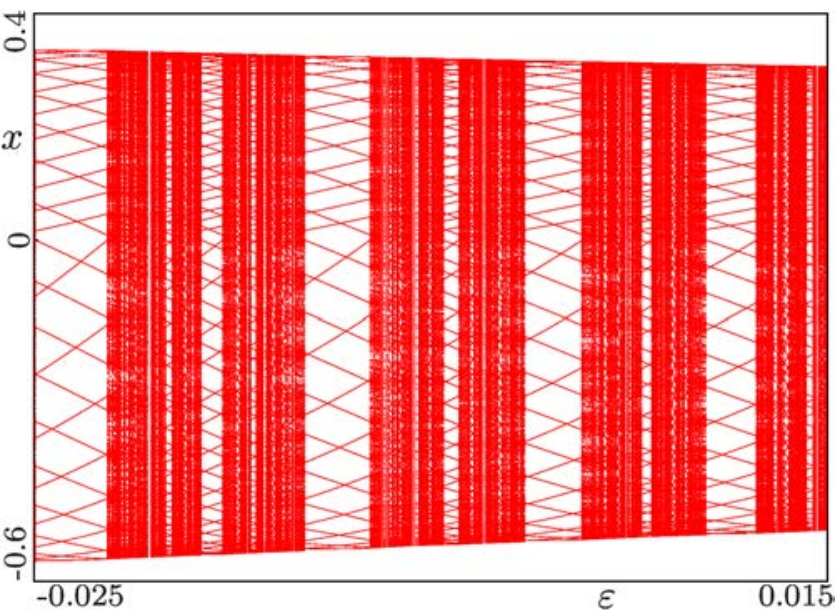

(a)

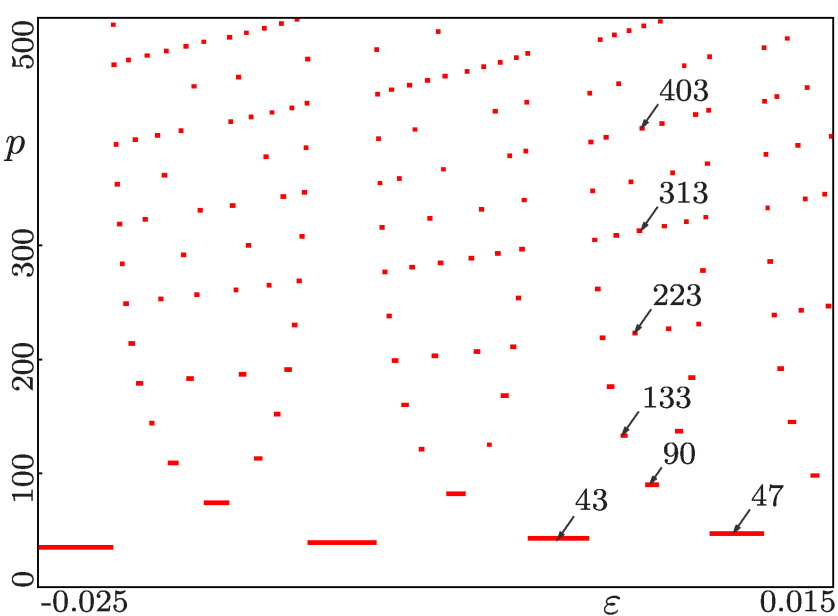

(b)

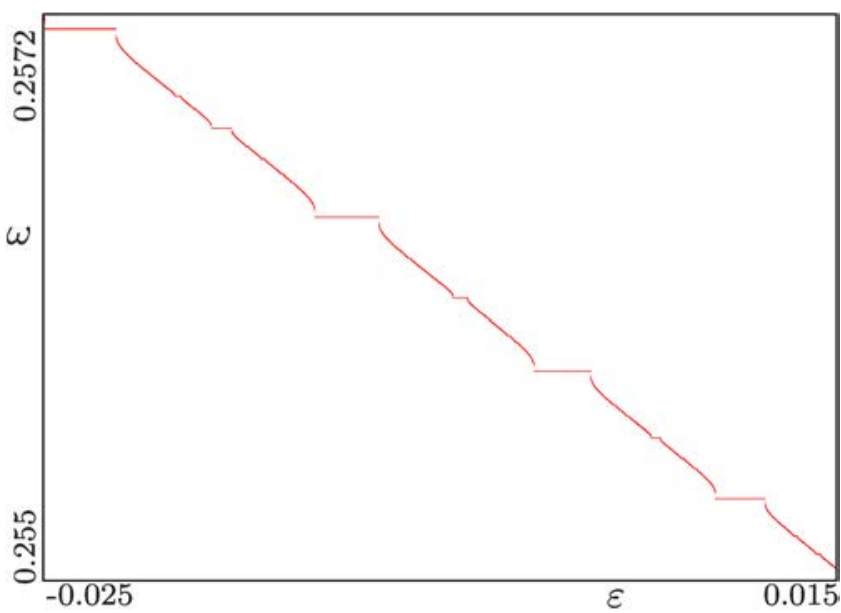

(c)

Fig. 2. Period adding structure arising as the parameters are varied along the path $\mathrm{A}$ indicated in Fig. 1(a) for $\tau_{\mathcal{R}}=$ $-0.1, \delta_{\mathcal{L}}=0.5, \delta_{\mathcal{R}}=1.8094, \mu=0.05$. (a) Bifurcation diagram, (b) period diagram and (c) rotation number $\omega$.

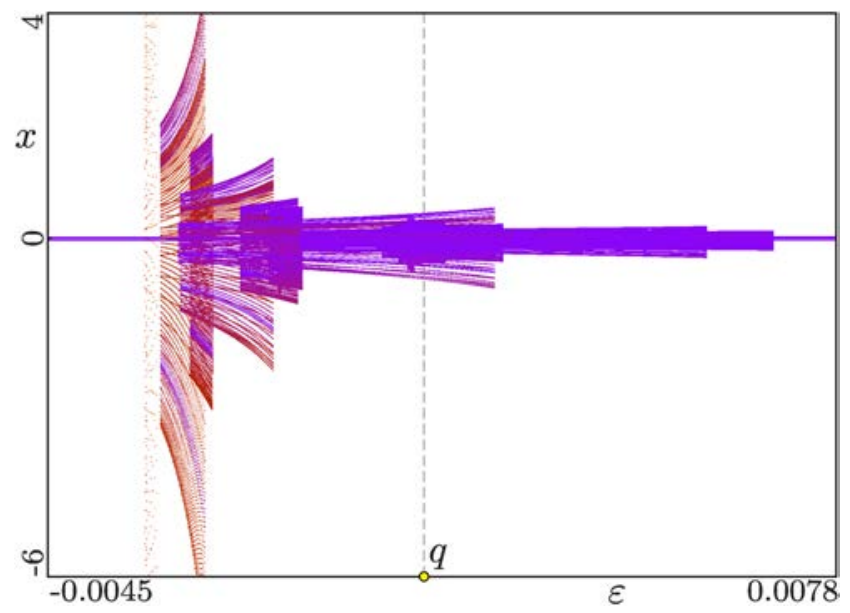

(a)

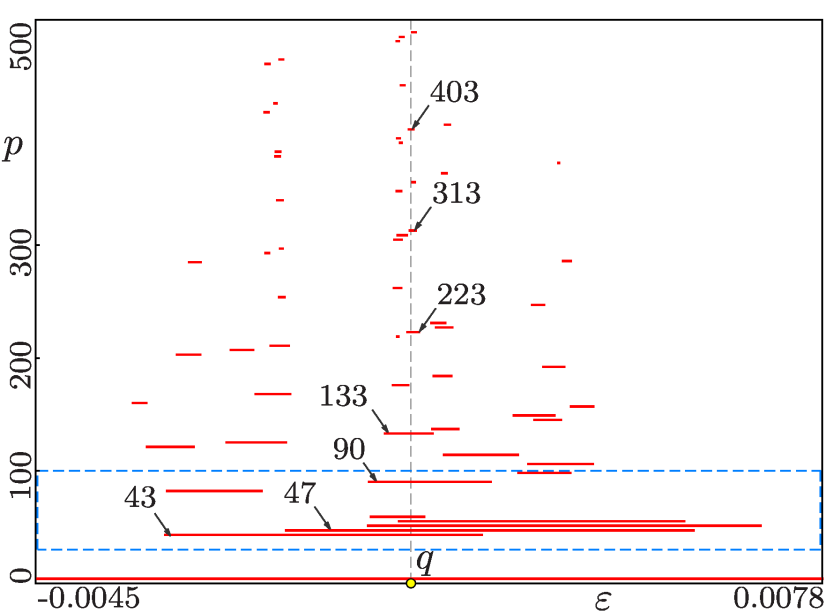

(b)

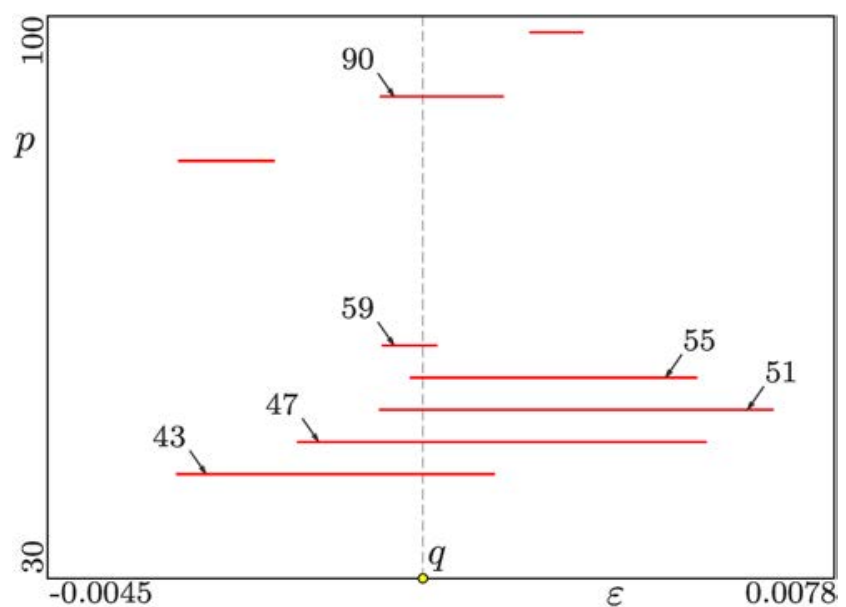

(c)

Fig. 3. (a) Bifurcation and (b) period diagrams along the parameter path B marked in Fig. 11(b), showing the appearance of coexisting attractors nested into each other. Periods of the stable cycles coexisting at the point $q$ are indicated. $\tau_{\mathcal{R}}=0.3, \delta_{\mathcal{L}}=0.5, \delta_{\mathcal{R}}=1.8094, \mu=0.05$. 


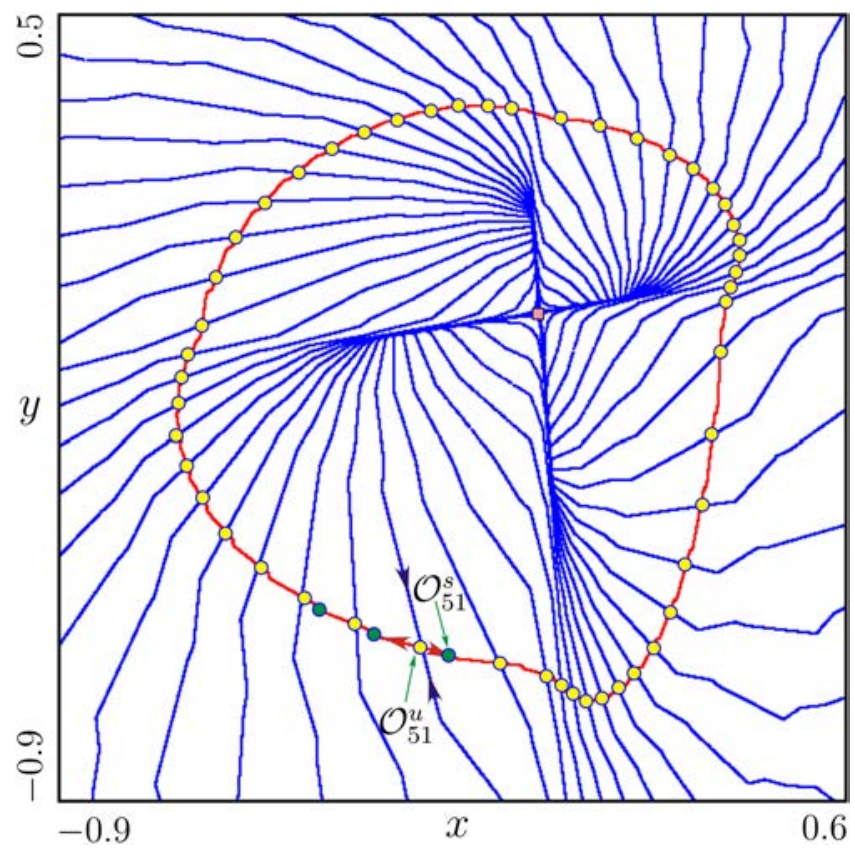

(a)

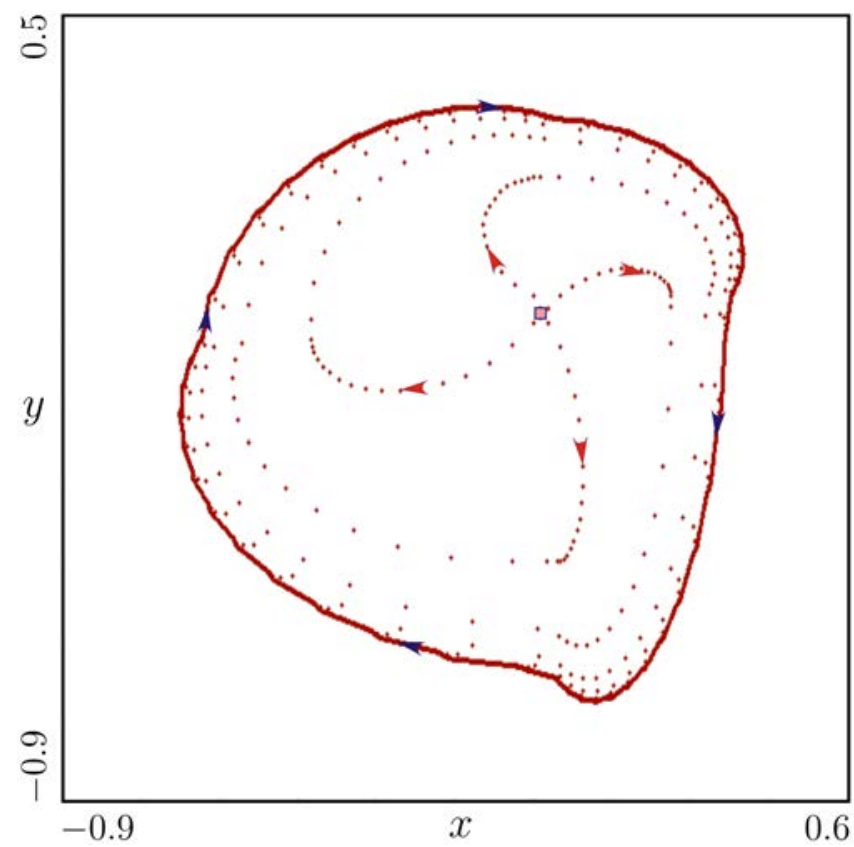

(b)

Fig. 4. Transition to quasiperiodic dynamics occurring when the system leaves the phase-locked region $\mathcal{P}_{51}$ through a bordercollision fold bifurcation curve. (a) Phase portrait of the map (1) inside the phase-locked region $\mathcal{P}_{51}$ for the parameter values $\tau_{\mathcal{R}}=-0.021$ and $\tau_{\mathcal{L}}=-0.0969815$ marked with $a$ in Fig. 1(b). Attracting closed invariant curve formed by a saddlenode connection between a 51-saddle $\mathcal{O}_{51}^{u}$ and the attracting 51-node $\mathcal{O}_{51}^{s}$. (b) The attracting closed invariant curve with quasiperiodic dynamics that arises through a border-collision fold bifurcation when the system leaves the phase-locked region $\mathcal{P}_{51}$. This phase portrait illustrates the closed invariant curve existing at the point $b$ [see Fig. [1 b)] with $\tau_{\mathcal{R}}=-0.0218$, $\tau_{\mathcal{L}}=-0.0969815$

The next question arising here is whether the coexisting stable cycles belong to closed invariant curves. In fact, with increasing $\tau_{\mathcal{R}}$ some of the stable closed invariant curves previously existing in the regions belonging to the period adding structure are destroyed. The mechanisms leading to their destruction may be related to an interplay of bordercollision fold and homoclinic bifurcations (see, for instance [Zhusubalivev et al., 2006]) and are beyond the scope of the present paper.

Therefore, a possible mechanism leading to the appearance of the specific kind of multistability we are interested in, namely coexistence of closed invariant curves nested into each other, is related to the deformation of a period adding structure characterized by the following two conditions:

(1) a number of periodicity regions involved in the bifurcations structure do already overlap;

(2) the associated closed invariant curves are still not destroyed.

This may lead to a quite intricate structure in the state space, as discussed in the next section.

\section{Nested Invariant Curves}

To illustrate the complexity of the structures resulting from the overlap of the regions originating from the period adding structure, let us consider the dynamics of map (11) at the parameter point $q$ given by $\tau_{\mathcal{L}}=-0.39024, \tau_{\mathcal{R}}=0.3$ with the same values of $\delta_{\mathcal{L} / \mathcal{R}}$ as considered before and $\mu>0$. As one can see in Fig. 1] several regions overlap at this point. Indeed, numerical experiments suggest that for these parameter values the map (1) demonstrates 11 coexisting stable cycles

$$
\begin{aligned}
\mathcal{O}_{4}^{s}, \mathcal{O}_{43}^{s}, \mathcal{O}_{47}^{s}, \mathcal{O}_{51}^{s}, \mathcal{O}_{55}^{s}, \mathcal{O}_{59}^{s}, \\
\mathcal{O}_{90}^{s}, \mathcal{O}_{133}^{s}, \mathcal{O}_{223}^{s}, \mathcal{O}_{313}^{s}, \mathcal{O}_{403}^{s} .
\end{aligned}
$$

The cycles listed in (4) and their basins of attraction are shown in Fig. 5(a) and the magnifications are presented in Figs. 5(b) and 5(c). Throughout this paper, the basin of attraction of an attractor $\mathcal{A}$ (which may be a cycle or a closed invariant curve) is denoted by $\mathcal{B}(\mathcal{A})$. As one can see in Figs. 5(b) and 5(c), some of the boundaries of the basins have a quite complicated structure, while 


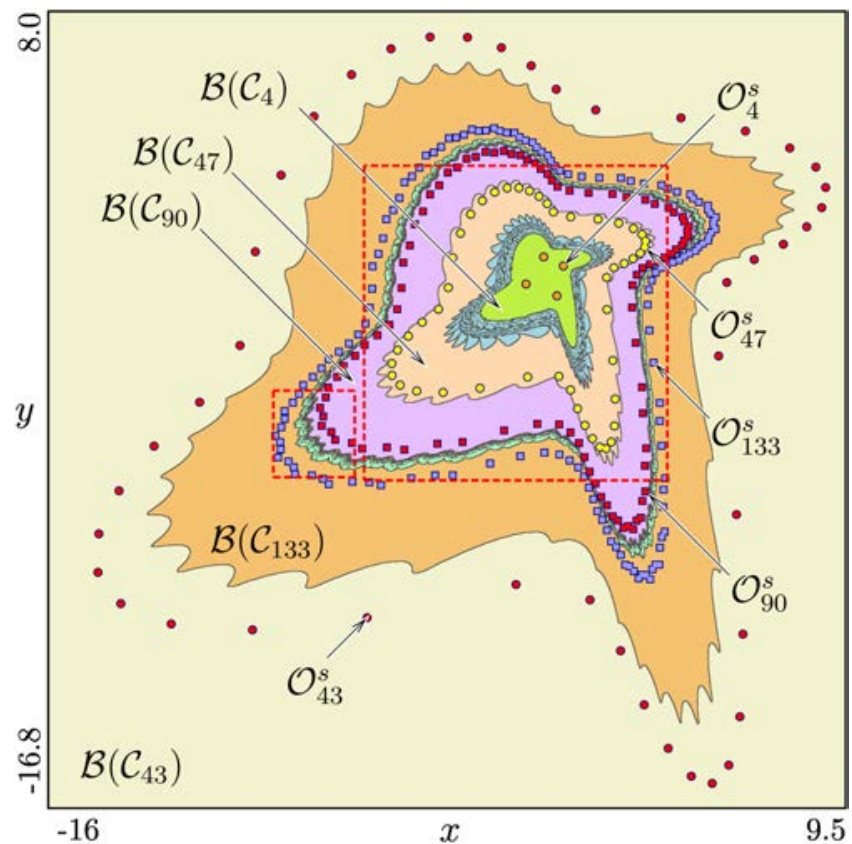

(a)

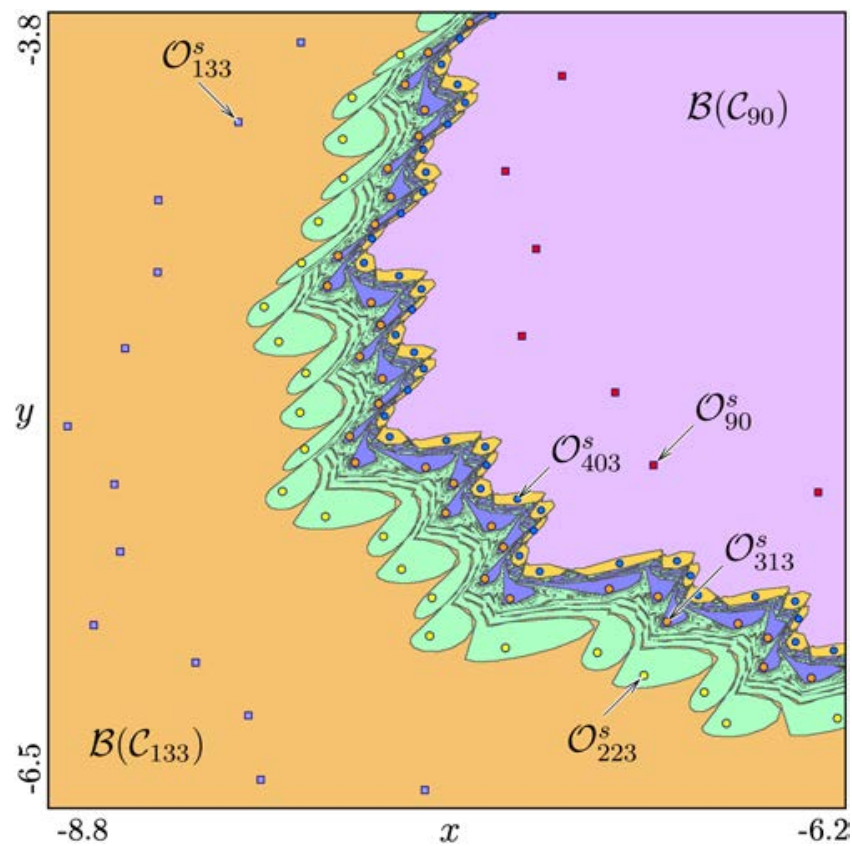

(c)

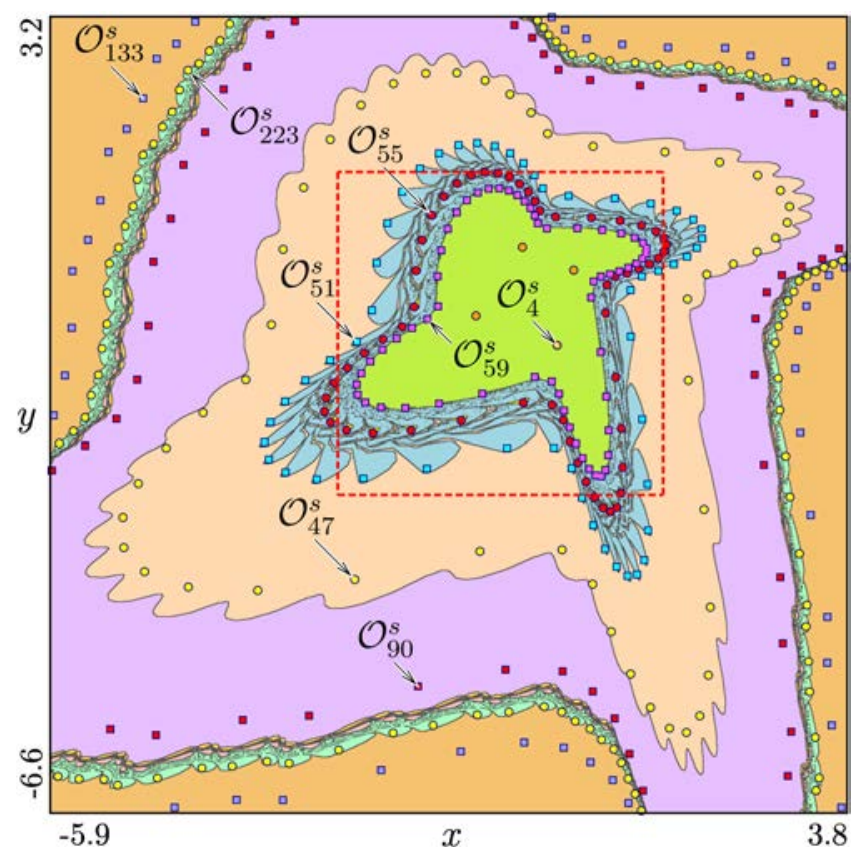

(b)

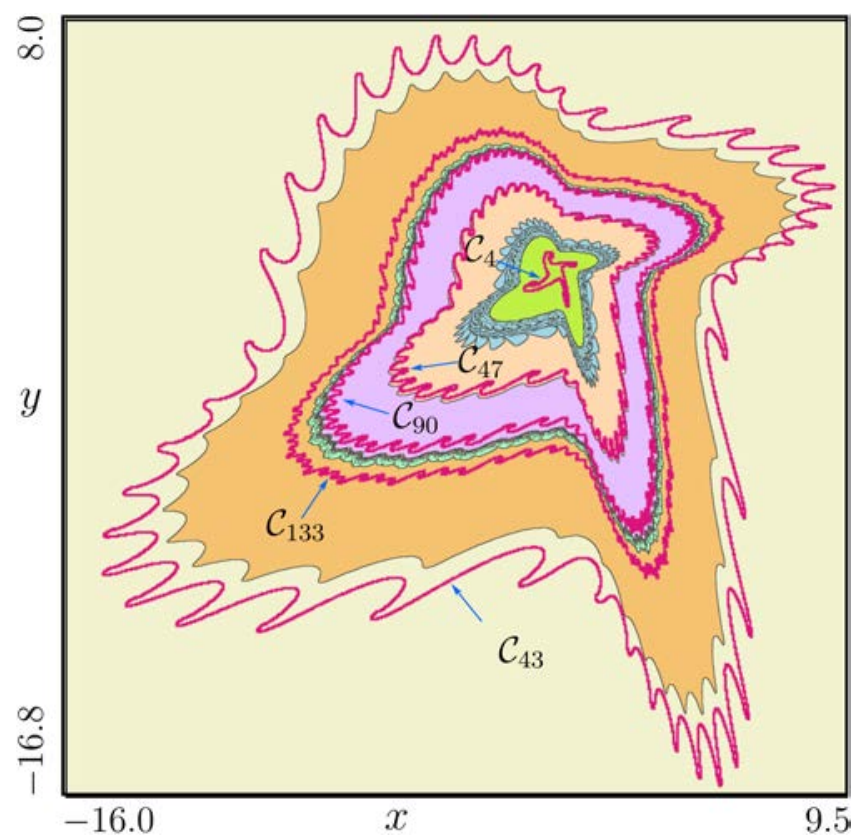

(d)

Fig. 5. Overview of the basins of attraction for coexisting cycles listed in (4). (a) Overall structure of the phase space. (b) and (c) Magnifications of the regions marked in (a). (d) Attracting nested closed invariant curves $\mathcal{C}_{4}, \mathcal{C}_{47}, \mathcal{C}_{90}, \mathcal{C}_{133}$, and $\mathcal{C}_{43}$. 
the shapes of the other boundaries are quite simple. As discussed below, this follows from the fact that among the cycles listed in (41), the cycles $\mathcal{O}_{4}^{s}, \mathcal{O}_{43}^{s}$, $\mathcal{O}_{47}^{s}, \mathcal{O}_{90}^{s}$, and $\mathcal{O}_{133}^{s}$ at the considered parameter values belong to nested attracting closed invariant curves $\mathcal{C}_{4}, \mathcal{C}_{43}, \mathcal{C}_{47}, \mathcal{C}_{90}$, and $\mathcal{C}_{133}$, respectively [see Fig. [5(d)], while the closed invariant curves involving the cycles $\mathcal{O}_{51}^{s}, \mathcal{O}_{55}^{s}, \mathcal{O}_{59}^{s}, \mathcal{O}_{223}^{s}, \mathcal{O}_{313}^{s}, \mathcal{O}_{403}^{s}$ are already destroyed.

In order to explain the formation of closed invariant curves existing at the point $q$ and their basins of attraction, let us now examine the phase portraits in Fig. 5 in more details, taking into considerations not only stable but also unstable cycles and their manifolds.

Figure [6)(a) shows a magnification of the part of the phase plane outlined by a rectangle in Fig. 5 (b) around the repelling focus fixed point. In this part we observe four attracting cycles, namely, $\mathcal{O}_{4}^{s}, \mathcal{O}_{51}^{s}$, $\mathcal{O}_{55}^{s}$, and $\mathcal{O}_{59}^{s}$.

As illustrated in Fig. 6(b), the attracting closed invariant curve $\mathcal{C}_{4}$ contains two 4 -cycles, a saddle $\mathcal{O}_{4}^{u}=\bigcup_{i=1}^{4} C_{4, i}^{u}$ and a stable focus $\mathcal{O}_{4}^{s}=\bigcup_{i=1}^{4} \mathcal{O}_{4, i}^{s}$, where $\mathcal{O}_{4, i}^{s / u}$ are periodic points, and the saddle-focus

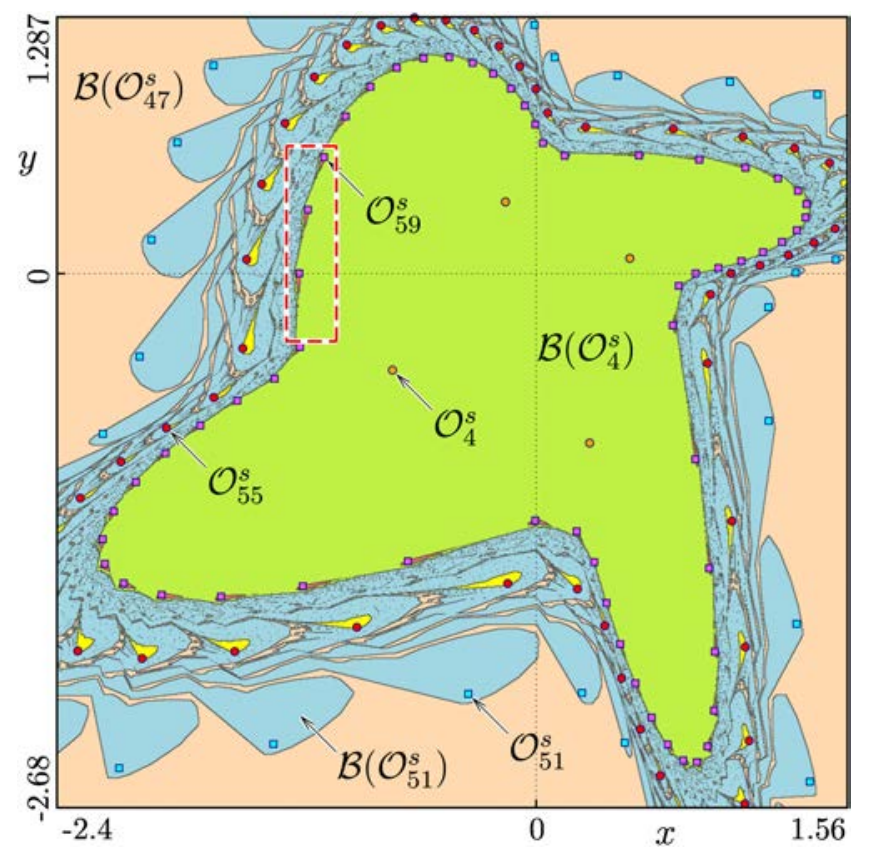

(a) connection is formed by the unstable manifolds of the saddle cycle $\mathcal{O}_{4}^{u}$. This stable, as well as the unstable manifold of $\mathcal{O}_{4}^{u}$ consists of two branches, separated by $\mathcal{O}_{4}^{u}$, and referred to in the following as $\mathcal{W}_{ \pm}^{s / u}\left(\mathcal{O}_{4}^{u}\right)=\bigcup_{i=1}^{4} \mathcal{W}_{ \pm}^{s / u}\left(\mathcal{O}_{4, i}^{u}\right)$. Recall that the manifolds $\mathcal{W}_{ \pm}^{s}$ and $\mathcal{W}_{ \pm}^{u}$ are invariant for $F$ in Eq. (1), while for each $i$ the set $\mathcal{W}_{ \pm}^{s / u}\left(\mathcal{O}_{4, i}^{u}\right)$ is invariant with respect to the fourth iterate $F^{4}$ Kuznetsov, 2004; Agliari et al., 2005.

As one can see in Fig. 6(b), the branch $\mathcal{W}_{-}^{s}\left(\mathcal{O}_{4}^{u}\right)$ of the stable manifolds of the saddle 4-cycle $\mathcal{O}_{4}^{u}$ issues from the repelling focus fixed point. The other branch $\mathcal{W}_{+}^{s}\left(\mathcal{O}_{4}^{u}\right)$ originates from a chaotic saddle $\mathcal{S}_{\mathrm{ch}}^{1}$, which serves as the boundary between the basins of attraction $\mathcal{B}\left(\mathcal{C}_{4}\right), \mathcal{B}\left(\mathcal{C}_{47}\right)$ of the closed invariant curves $\mathcal{C}_{4}$ and $\mathcal{C}_{47}$, respectively. The basins of the other stable cycles $\mathcal{O}_{51}^{s}, \mathcal{O}_{55}^{s}$, and $\mathcal{O}_{59}^{s}$ are "embedded" into the basins of the closed invariant curves $\mathcal{C}_{4}$ and $\mathcal{C}_{47}$, as illustrated in Figs. 7(a) and $7(\mathrm{~b})$, showing the structure of the phase space in the neighborhood of the chaotic saddle $\mathcal{S}_{\mathrm{ch}}^{1}$.

In Fig. 7(a), which corresponds to the region outlined in Figs. 6(a) and 6(b), one can see a

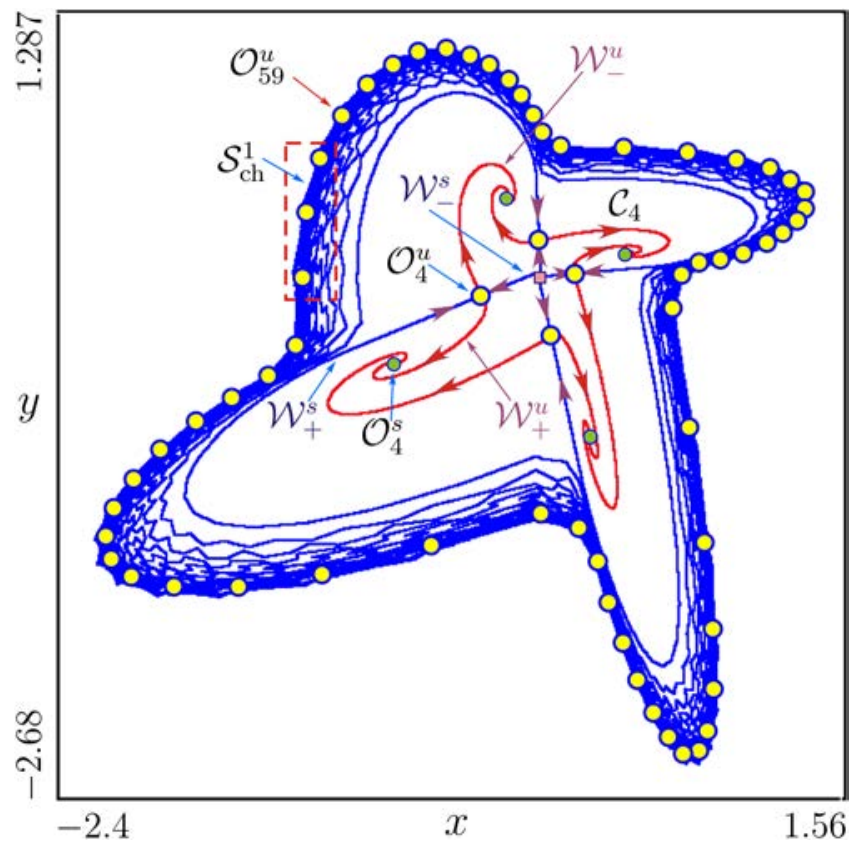

(b)

Fig. 6. Phase portrait of map (1) in the region around the attracting closed invariant curve $\mathcal{C}_{4}$. (a) The basin of attraction of $\mathcal{C}_{4}$ and the basins $\mathcal{B}\left(\mathcal{O}_{59}^{s}\right), \mathcal{B}\left(\mathcal{O}_{55}^{s}\right), \mathcal{B}\left(\mathcal{O}_{51}^{s}\right)$ issuing from the chaotic saddle $\mathcal{S}_{\text {ch }}^{1}$. (b) The closed invariant curve $\mathcal{C}_{4}$ formed by the stable 4 -focus $\mathcal{O}_{4}^{s}$, the 4-saddle $\mathcal{O}_{4}^{u}$ and its unstable manifold $\mathcal{W}_{ \pm}^{u}\left(\mathcal{O}_{4}^{u}\right)$. Additionally, the stable manifold $\mathcal{W}_{ \pm}^{s}\left(\mathcal{O}_{4}^{u}\right)$ of $\mathcal{O}_{4}^{u}$ is shown, issuing on one side (the branch $\mathcal{W}_{-}^{s}\left(\mathcal{O}_{4}^{u}\right)$ ) from the repelling fixed point and on the other side $\left(\right.$ the branch $\mathcal{W}_{+}^{s}\left(\mathcal{O}_{4}^{u}\right)$ ) from the chaotic saddle $\mathcal{S}_{\mathrm{ch}}^{1}$. The region marked by a rectangle in (a) and (b) is shown enlarged in Fig. 7. 


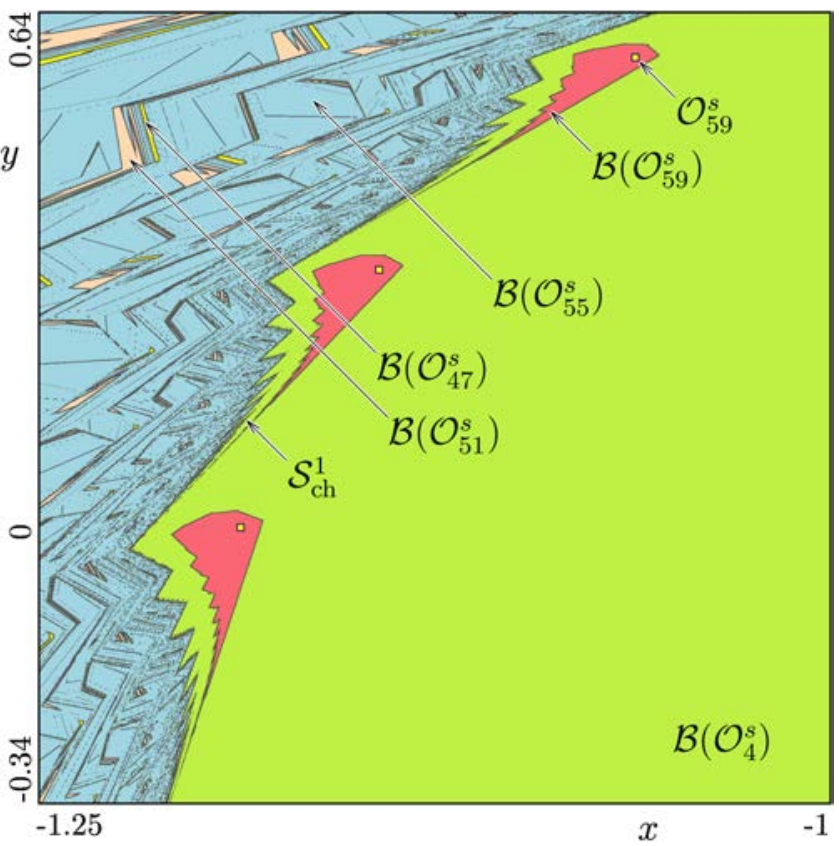

(a)

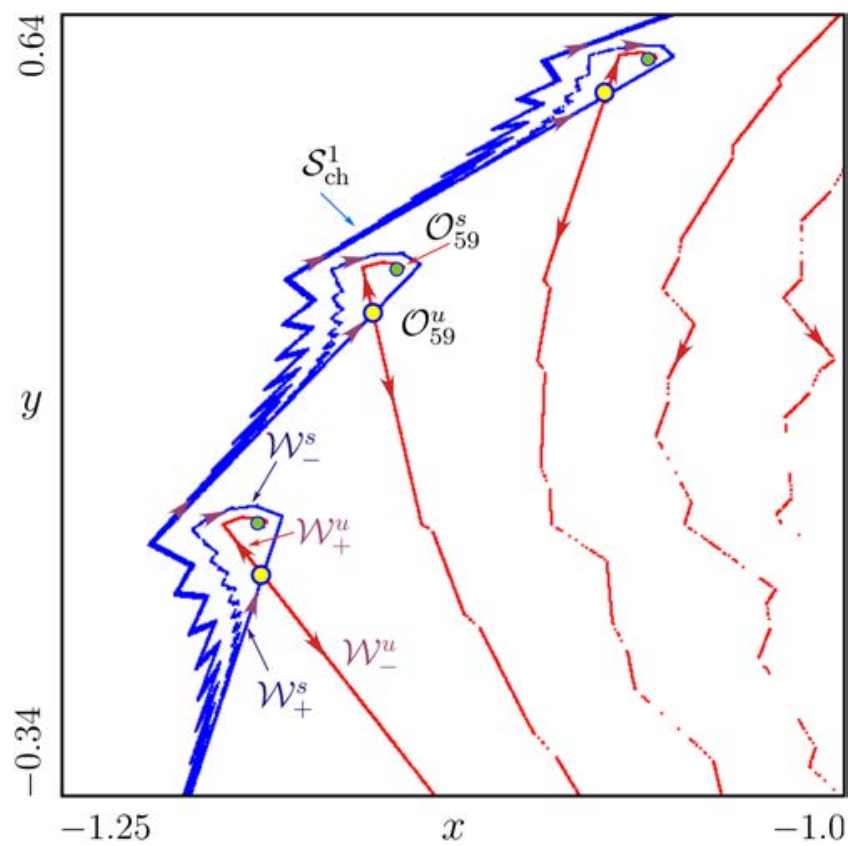

(b)

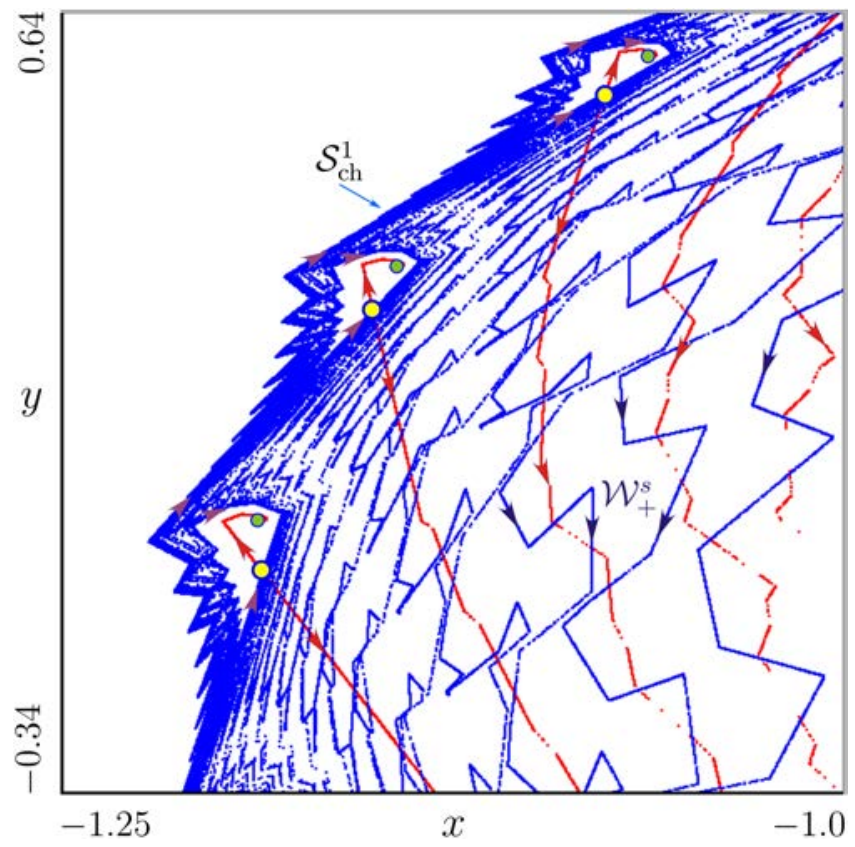

(c)

Fig. 7. Magnification of the region marked by a rectangle in Fig. 6 (a) Basin of attraction $\mathcal{B}\left(\mathcal{O}_{59}^{s}\right)$ embedded into the basin $\mathcal{B}\left(\mathcal{C}_{4}\right)$ and converging to the chaotic saddle $\mathcal{S}_{\mathrm{ch}}^{1}$. (b) Phase portrait illustrating the boundary of the basin of attraction for a stable 59-cycle $\mathcal{O}_{59}^{s}$. The branch $\mathcal{W}_{+}^{u}\left(\mathcal{O}_{59}^{u}\right)$ of the unstable manifold of $\mathcal{O}_{59}^{u}$ converges to the 59 -focus $\mathcal{O}_{59}^{s}$, while the branch $\mathcal{W}_{-}^{u}\left(\mathcal{O}_{59}^{u}\right)$ converges to the closed invariant curve $\mathcal{C}_{4}$. Both branches $\mathcal{W}_{ \pm}^{s}\left(\mathcal{O}_{59}^{u}\right)$ of the stable manifold of $\mathcal{O}_{59}^{u}$ issue from the chaotic saddle $\mathcal{S}_{\mathrm{ch}}^{1}$ and form the boundary of the basin of attraction for $\mathcal{O}_{59}^{s}$. (c) Phase portrait showing the heteroclinic intersections of the unstable $\mathcal{W}_{+}^{u}\left(\mathcal{O}_{59}^{u}\right)$ and stable $\mathcal{W}_{+}^{u}\left(\mathcal{O}_{4}^{u}\right)$ manifolds of the saddle 59- and 4-cycles, respectively. The unstable manifold $\mathcal{W}_{ \pm}^{u}\left(\mathcal{O}_{59}^{u}\right)$ of the 59 -saddle $\mathcal{O}_{59}^{u}$ and the branch $\mathcal{W}_{+}^{s}\left(\mathcal{O}_{4}^{u}\right)$ of the stable manifold of the 4-saddle $\mathcal{O}_{4}^{u}$ are shown. Its intersection with the unstable manifold of $\mathcal{O}_{59}^{u}$, i.e. $\mathcal{W}_{+}^{s}\left(\mathcal{O}_{4}^{u}\right) \cap \mathcal{W}_{+}^{u}\left(\mathcal{O}_{59}^{u}\right)$, forms a heteroclinic structure. 
magnification of the basin of attraction $\mathcal{B}\left(\mathcal{O}_{59}^{s}\right)$ of the stable 59-cycle $\mathcal{O}_{59}^{s}$. Figure 7 (b) shows the stable and unstable manifolds of the saddle 59-cycle separately. It is clearly visible that the stable 59cycle $\mathcal{O}_{59}^{s}$ is surrounded by the stable manifolds

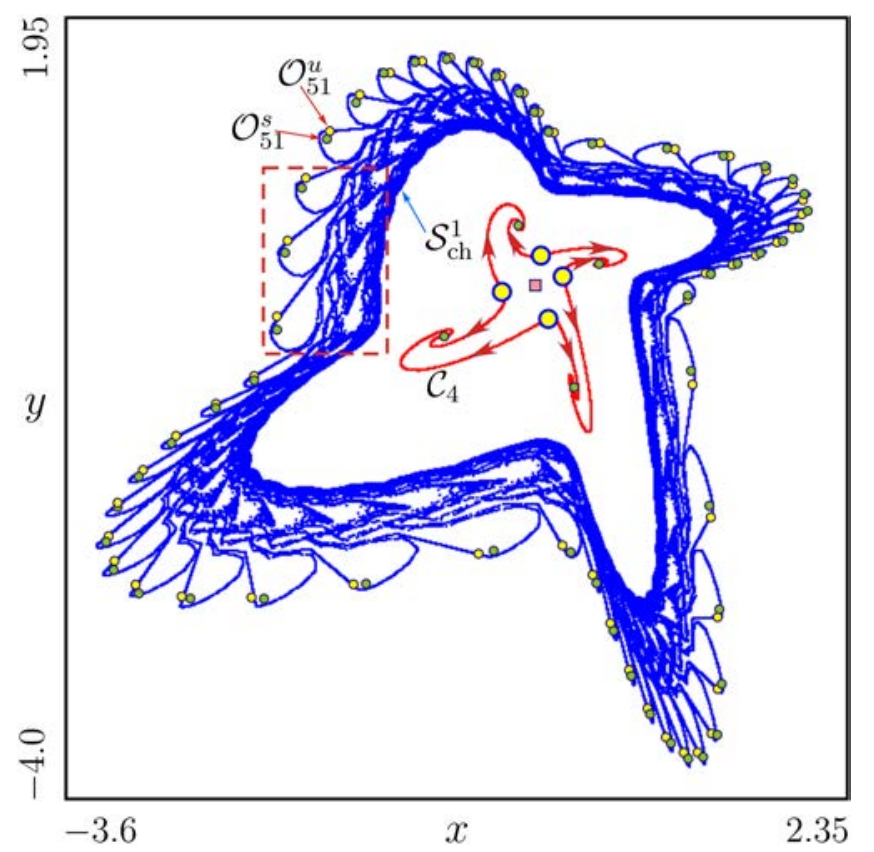

(a)

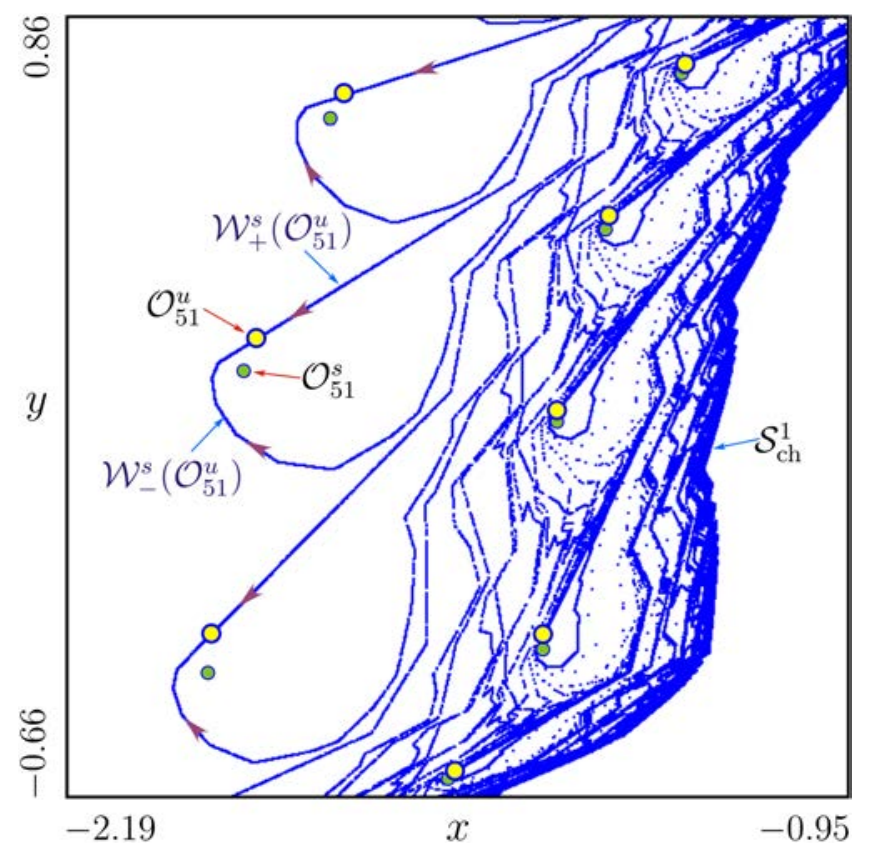

(c)
$\mathcal{W}_{ \pm}^{s}\left(\mathcal{O}_{59}^{u}\right)$ of the saddle 59-cycle $\mathcal{O}_{59}^{u}$. In this way, the stable manifolds of $\mathcal{O}_{59}^{u}$ form a boundary between the basins of attraction of $\mathcal{O}_{59}^{s}$ and $\mathcal{C}_{4}$. Furthermore, both branches of the stable manifold $\mathcal{W}_{ \pm}^{s}\left(\mathcal{O}_{59}^{u}\right)$ originate from the chaotic saddle $\mathcal{S}_{\mathrm{ch}}^{1}$ so that the basin

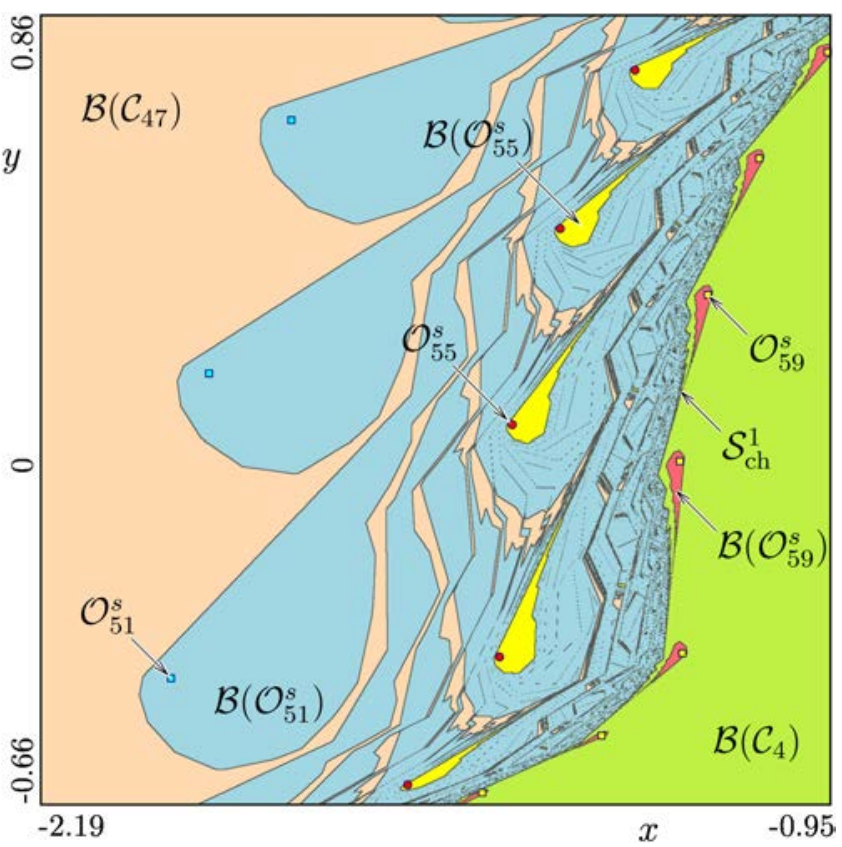

(b)

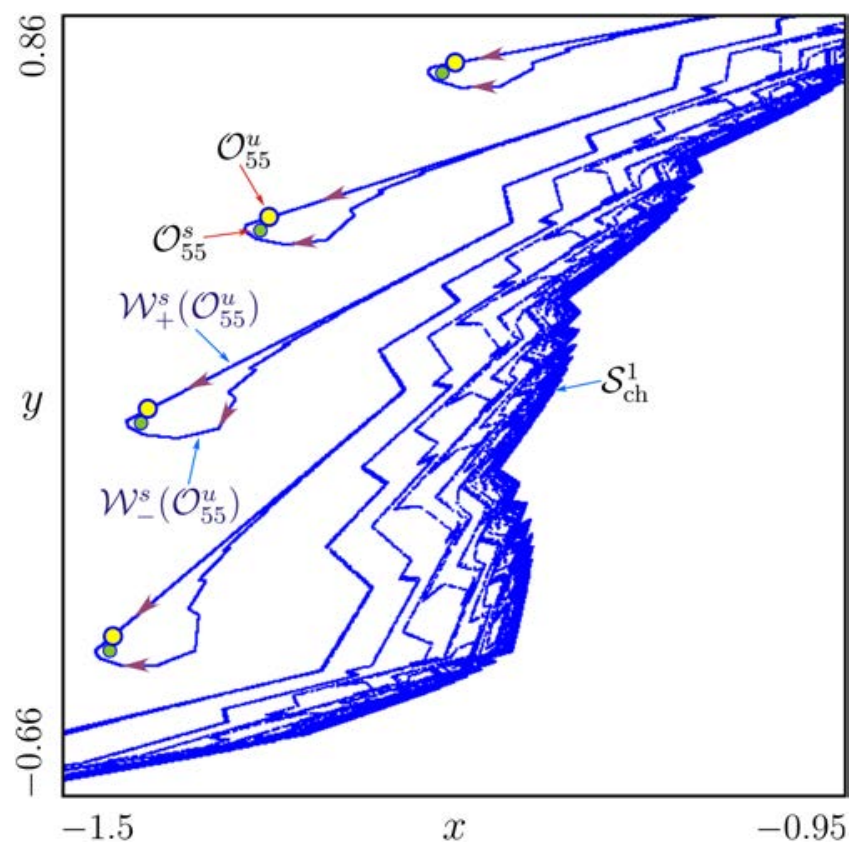

(d)

Fig. 8. (a) Structure of the phase space close to the boundary between the basins of the closed invariant curves $\mathcal{C}_{4}$ and $\mathcal{C}_{47}$. (b) Magnification of the region marked in (a), showing the basins of attraction $\mathcal{B}\left(\mathcal{C}_{4}\right), \mathcal{B}\left(\mathcal{O}_{59}^{s}\right), \mathcal{B}\left(\mathcal{O}_{55}^{s}\right), \mathcal{B}\left(\mathcal{O}_{51}^{s}\right)$, and $\mathcal{B}\left(\mathcal{C}_{47}\right)$ issuing from the chaotic saddle $\mathcal{S}_{\mathrm{ch}}^{1}$. (c) Phase portrait illustrating the boundary of the basin of attraction for a stable 51-cycle $\mathcal{O}_{51}^{s}$. (d) Boundary of the basin of attraction for a stable 55-cycle $\mathcal{O}_{55}^{s}$. 
of attraction $\mathcal{B}\left(\mathcal{O}_{59}^{s}\right)$ is embedded into the basin of attraction $\mathcal{B}\left(\mathcal{C}_{4}\right)$ of the closed invariant curve $\mathcal{C}_{4}$. Note that the branch $\mathcal{W}_{+}^{s}\left(\mathcal{O}_{4}^{u}\right)$ of the stable manifold of $\mathcal{O}_{4}^{u}$ originates from the chaotic saddle $\mathcal{S}_{\text {ch }}^{1}$ as well [see Fig. 6(a)]. Figure [7(c) shows this branch and the stable manifold of $\mathcal{O}_{59}^{u}$ in the neighborhood of $\mathcal{O}_{59}^{u}$ and the chaotic saddle $\mathcal{S}_{\mathrm{ch}}^{1}$. As illustrated in this figure, both branches intersect and form a heteroclinic structure which leads to a quite complex structure of the boundary of $\mathcal{B}\left(\mathcal{C}_{4}\right)$.

Outside the chaotic saddle $\mathcal{S}_{\mathrm{ch}}^{1}$, a similar structure is formed by the basins of attraction $\mathcal{B}\left(\mathcal{O}_{55}^{s}\right)$, $\mathcal{B}\left(\mathcal{O}_{51}^{s}\right)$ of the stable 55 - and 51-cycles $\mathcal{O}_{55}^{s}, \mathcal{O}_{51}^{s}$, as illustrated in Fig. 8(a). As one can see in Fig. 8(b), the basins $\mathcal{B}\left(\mathcal{O}_{55}^{s}\right)$ and $\mathcal{B}\left(\mathcal{O}_{51}^{s}\right)$ are embedded into the basin $\mathcal{B}\left(\mathcal{C}_{47}\right)$ of the closed invariant curve $\mathcal{C}_{47}^{s}$ described below in the same way as the basin $\mathcal{B}\left(\mathcal{O}_{59}^{s}\right)$ is embedded into the basin $\mathcal{B}\left(\mathcal{C}_{4}^{s}\right)$. Indeed, the basin $\mathcal{B}\left(\mathcal{O}_{51}^{s}\right)$ is confined by the stable manifold of the saddle 51-cycle $\mathcal{O}_{51}^{u}$ and both sets of the branches $\mathcal{W}_{ \pm}^{s}\left(\mathcal{O}_{51}^{u}\right)$ of this manifold originate from the chaotic saddle $\mathcal{S}_{\text {ch }}^{1}$ [see Fig. 8(c)]. Similarly, both branches $\mathcal{W}_{ \pm}^{s}\left(\mathcal{O}_{55}^{u}\right)$ of the stable manifold of the saddle $\mathcal{O}_{55}^{u}$ confining the basin $\mathcal{B}\left(\mathcal{O}_{55}^{s}\right)$ originate from $\mathcal{S}_{\text {ch }}^{1}$ as well [see Fig. 8 $8(\mathrm{~d})$ ].

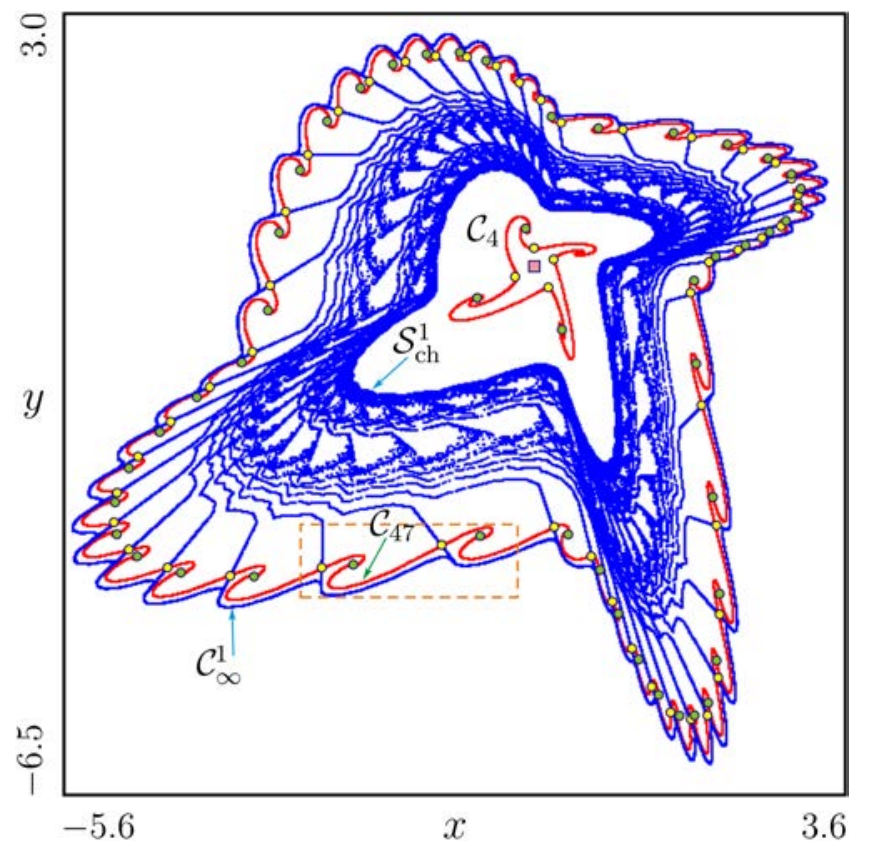

(a)
The closed invariant curve $\mathcal{C}_{47}$ is shown in Fig. 9(a). As indicated in the magnification presented in Fig. 9(b), $\mathcal{C}_{47}$ is formed by the stable focus $\mathcal{O}_{47}^{s}$, the saddle 47 -cycle $\mathcal{O}_{47}^{u}$ and its unstable manifold $\mathcal{W}_{ \pm}^{u}\left(\mathcal{O}_{47}^{u}\right)$. Note that the inner branch $\mathcal{W}_{+}^{s}\left(\mathcal{O}_{47}^{u}\right)$ of the stable manifold of $\mathcal{O}_{47}^{u}$ originates from the chaotic saddle $\mathcal{S}_{\mathrm{ch}}^{1}$ and intersects transversally the unstable manifolds of the saddle 51- and 55 -cycles $\mathcal{O}_{55}^{s}, \mathcal{O}_{51}^{s}$, forming a heteroclinic structure [see Fig. 9(a)]. The other branch $\mathcal{W}_{-}^{s}\left(\mathcal{O}_{47}^{u}\right)$ of this manifold issues from the repelling closed invariant curve $\mathcal{C}_{\infty}^{1}$ located outside $\mathcal{C}_{47}$. This repelling invariant curve separates the basin of attraction $\mathcal{B}\left(\mathcal{C}_{47}\right)$ from the basin of the next closed invariant curve $\mathcal{C}_{90}$.

The attracting closed invariant curve $\mathcal{C}_{90}$ is shown in Fig. 10] As indicated in the magnification presented in Fig. 10(b), it is formed by the stable focus $\mathcal{O}_{90}^{s}$, the saddle 90 -cycle $\mathcal{O}_{90}^{u}$ and its unstable manifolds $\mathcal{W}_{ \pm}^{u}\left(\mathcal{O}_{90}^{u}\right)$.

As one can see in Figs. 10(a) and 10(b), the branch $\mathcal{W}_{-}^{s}\left(\mathcal{O}_{90}^{u}\right)$ of the stable manifold of $\mathcal{O}_{90}^{u}$ originates from the repelling invariant curve $\mathcal{C}_{\infty}^{1}$. The other branch $\mathcal{W}_{+}^{s}\left(\mathcal{O}_{90}^{u}\right)$ issues from the chaotic saddle $\mathcal{S}_{\text {ch }}^{2}$ surrounding $\mathcal{C}_{90}$. The structure of the

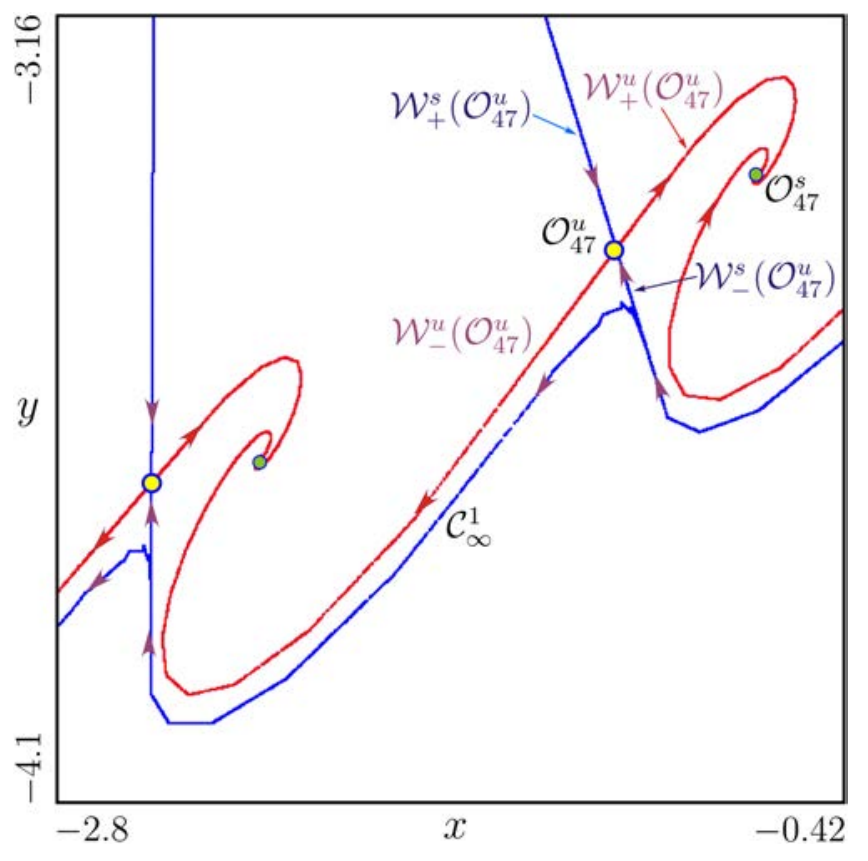

(b)

Fig. 9. (a) Attracting closed invariant curve $\mathcal{C}_{47}$ embedded into the region delineated by the chaotic saddle $\mathcal{S}_{\text {ch }}^{1}$ and the repelling invariant curve $\mathcal{C}_{\infty}^{1}$. (b) Magnified part of the phase portrait that is outlined by the rectangle in (a). Closed invariant curve $\mathcal{C}_{47}$ formed by the stable 47 -focus $\mathcal{O}_{47}^{s}$, the 47 -saddle $\mathcal{O}_{47}^{u}$ and its unstable manifolds $\mathcal{W}_{ \pm}^{u}\left(\mathcal{O}_{59}^{u}\right)$. The branch $\mathcal{W}_{+}^{s}\left(\mathcal{O}_{4}^{u}\right)$ of the stable manifold of $\mathcal{O}_{4}^{u}$ issues from the chaotic saddle $\mathcal{S}_{\mathrm{ch}}^{1}$, the other branch $\mathcal{W}_{-}^{s}\left(\mathcal{O}_{4}^{u}\right)$ from the repelling closed invariant curve $\mathcal{C}_{\infty}^{1}$. 


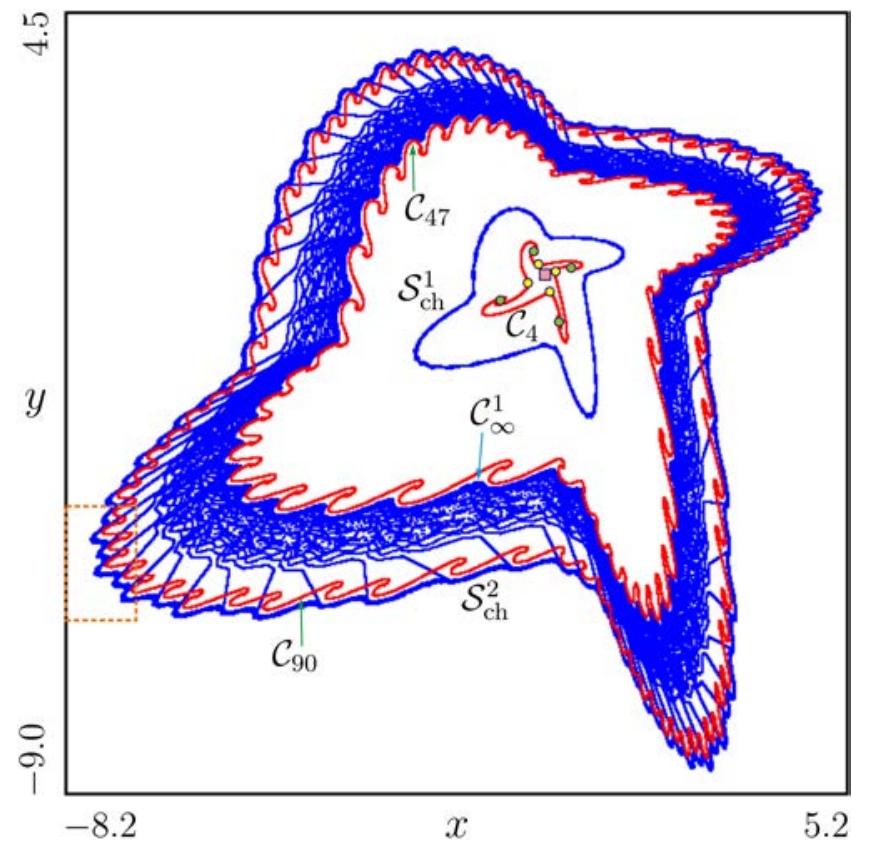

(a)

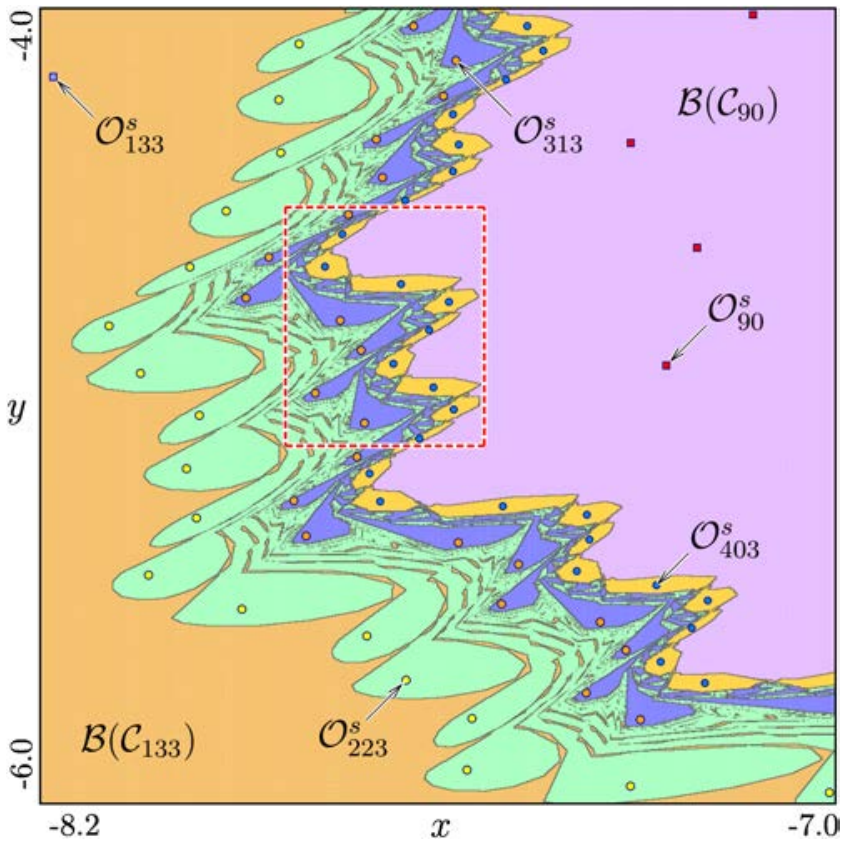

(c)

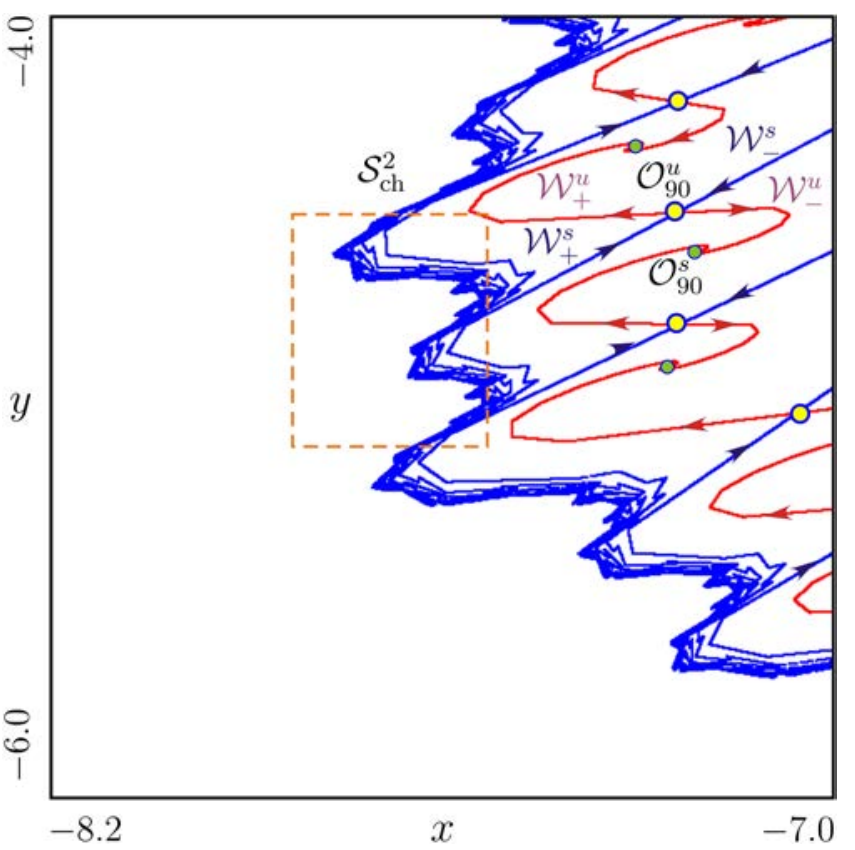

(b)

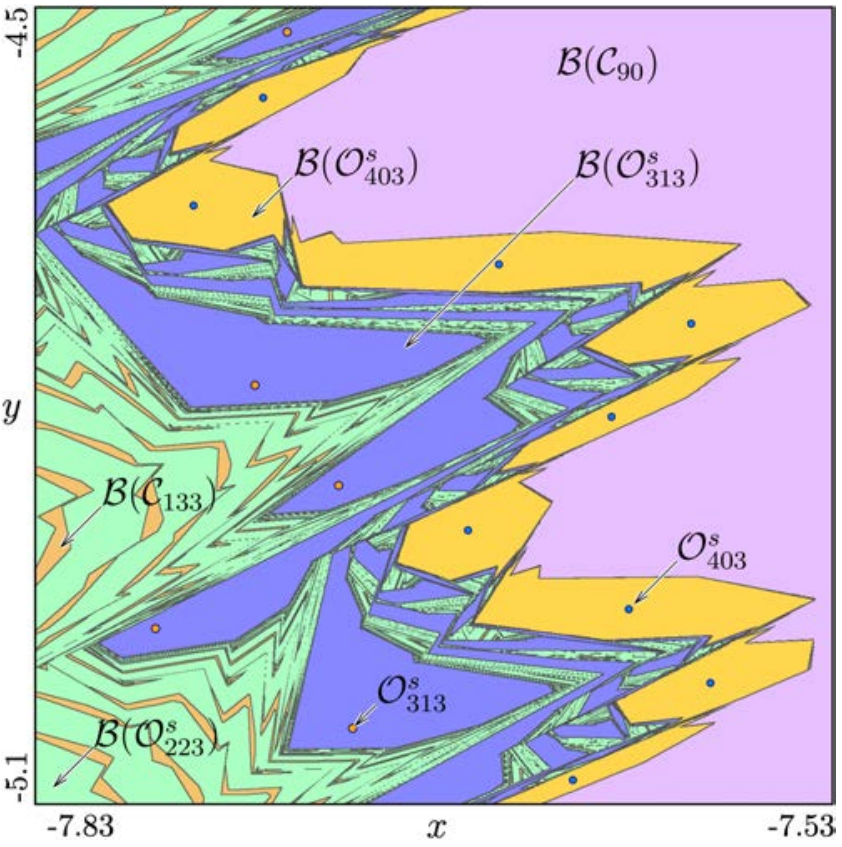

(d)

Fig. 10. (a) Phase portrait showing the boundary of the basin of attraction for the stable closed invariant curve $\mathcal{C}_{90}$. (b) Magnifications of the regions marked in (a). Closed invariant curve $\mathcal{C}_{90}$ formed by the stable 90 -focus $\mathcal{O}_{90}^{s}$, the 90 -saddle $\mathcal{O}_{90}^{u}$ and its unstable manifolds. One branch of the stable manifold of $\mathcal{O}_{90}^{u}$ issues from the repelling closed invariant curve $\mathcal{C}_{\infty}^{1}$, the other branch from the chaotic saddle $\mathcal{S}_{\text {ch }}^{2}$. (c) The same region as in (b), the basins of attraction $\mathcal{B}\left(\mathcal{C}_{90}\right), \mathcal{B}\left(\mathcal{O}_{223}^{s}\right)$, $\mathcal{B}\left(\mathcal{O}_{313}^{s}\right), \mathcal{B}\left(\mathcal{O}_{403}^{s}\right)$, and $\mathcal{B}\left(\mathcal{C}_{133}\right)$ issuing from the chaotic saddle $\mathcal{S}_{\mathrm{ch}}^{2}$ are shown. (d) Magnifications of the regions marked in (c). 


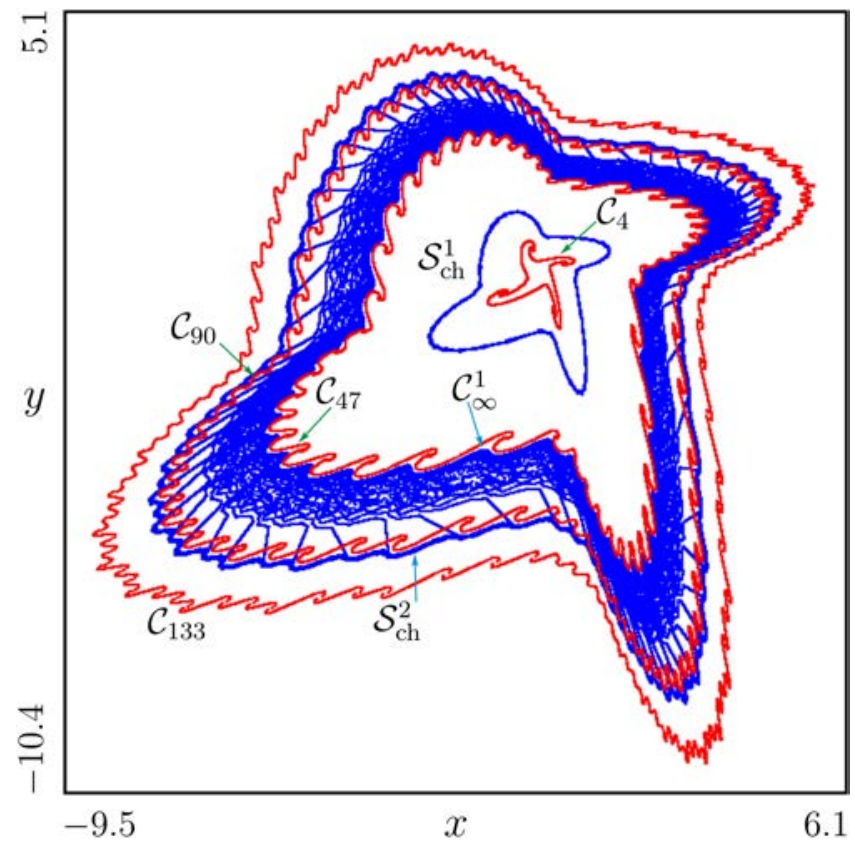

(a)

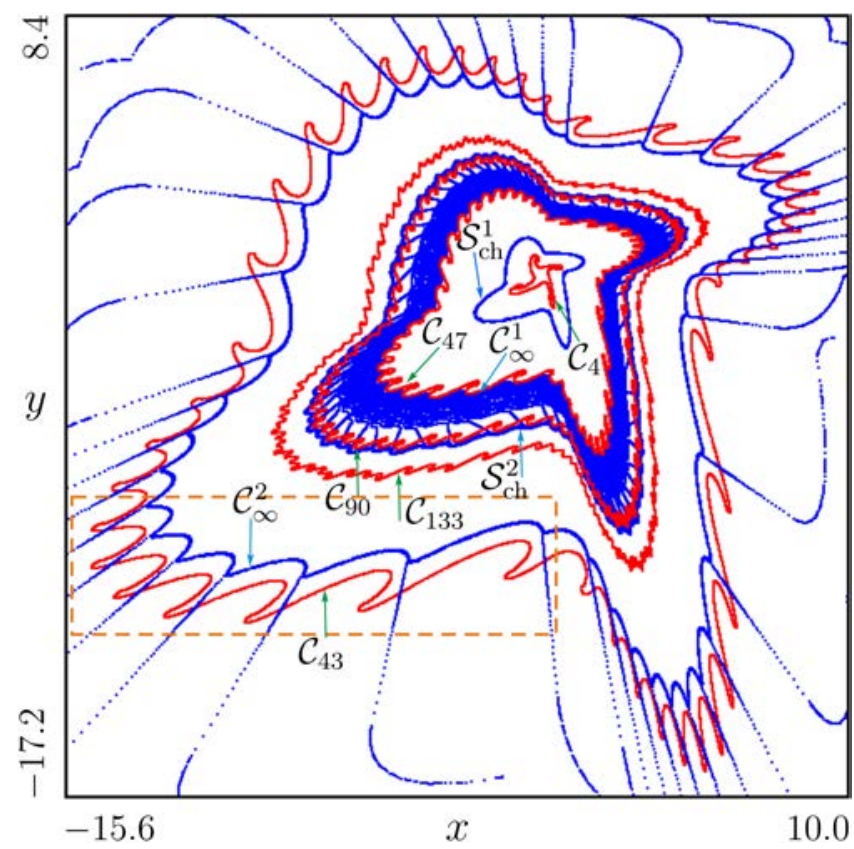

(b)

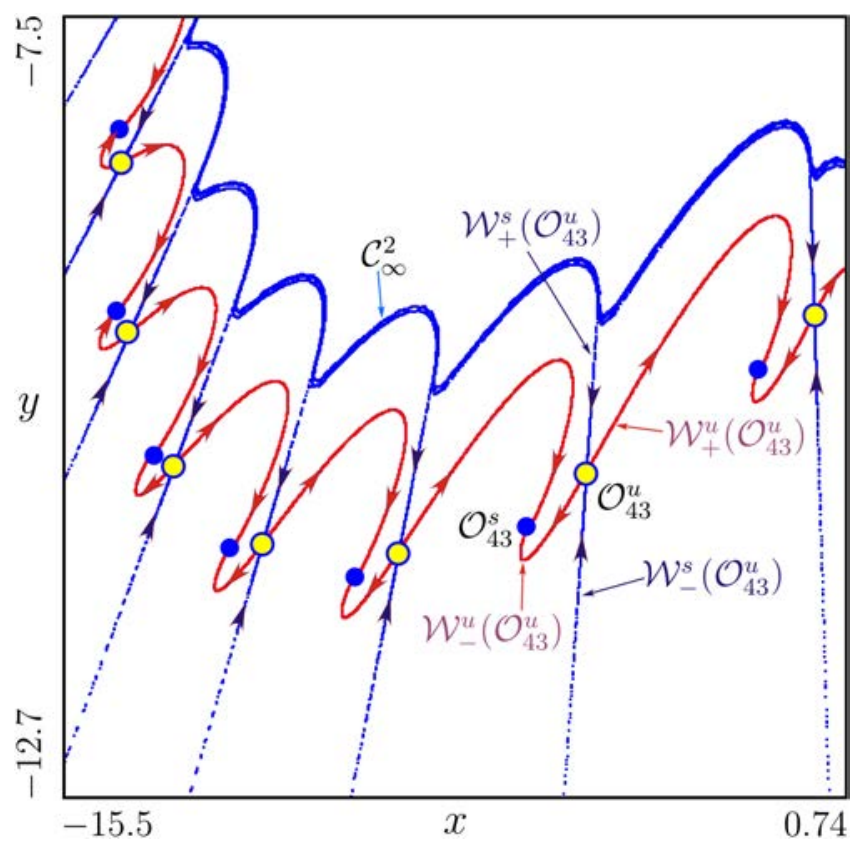

(c)

Fig. 11. (a) Phase portrait showing the coexistence of the stable closed invariant curve $\mathcal{C}_{133}$ and the stable closed curves $\mathcal{C}_{90}$, $\mathcal{C}_{47}$ and $\mathcal{C}_{4}$ nested into each other. (b) Overall structure of the phase portrait for the coexisting attracting closed invariant curves $\mathcal{C}_{43}, \mathcal{C}_{133}, \mathcal{C}_{90}, \mathcal{C}_{47}$ and $\mathcal{C}_{4}$ [see also Fig. [5.(d)]. (c) Magnification of the rectangle marked in (b), showing the attracting closed invariant curve $\mathcal{C}_{43}$ and the repelling closed invariant curve $\mathcal{C}_{\infty}^{2}$ separating the basins $\mathcal{C}_{133}$ and $\mathcal{C}_{43}$ from each other. The branch $\mathcal{W}_{+}^{s}\left(\mathcal{O}_{43}^{u}\right)$ issues from $\mathcal{C}_{\infty}^{2}$.

phase space close to $\mathcal{S}_{\mathrm{ch}}^{2}$ is illustrated in Figs. 10(c) and 10(d). As one can see, $\mathcal{S}_{\text {ch }}^{2}$ separates the basin $\mathcal{B}\left(\mathcal{C}_{90}\right)$ from the basin $\mathcal{B}\left(\mathcal{C}_{133}\right)$ of the closed invariant curve $\mathcal{C}_{133}$ and additionally, from the basins of the stable cycles $\mathcal{O}_{223}^{s}, \mathcal{O}_{313}^{s}$ and $\mathcal{O}_{403}^{s}$. Similar to the structure close to the chaotic saddle $\mathcal{S}_{\mathrm{ch}}^{1}$, the basins $\mathcal{B}\left(\mathcal{O}_{223}^{s}\right), \mathcal{B}\left(\mathcal{O}_{313}^{s}\right)$ and $\mathcal{B}\left(\mathcal{O}_{403}^{s}\right)$ of these cycles are embedded into the basin $\mathcal{B}\left(\mathcal{C}_{133}\right)$ of the 
closed invariant curve $\mathcal{C}_{133}$. Indeed, these basins are confined by the stable manifolds of the unstable cycles $\mathcal{O}_{223}^{u}, \mathcal{O}_{313}^{u}$ and $\mathcal{O}_{403}^{u}$, respectively, and both branches of the stable manifolds of these cycles originate from $\mathcal{S}_{\text {ch }}^{2}$.

As illustrated in Fig. 11, outside the chaotic saddle $\mathcal{S}_{\text {ch }}^{2}$ there are two more attracting closed invariant curves, namely $\mathcal{C}_{133}$ (formed by the stable focus $\mathcal{O}_{133}^{s}$, the saddle $\mathcal{O}_{133}^{u}$ and its unstable manifold $\mathcal{W}_{ \pm}^{u}\left(\mathcal{O}_{133}^{u}\right)$ ) and $\mathcal{C}_{43}$ (containing the stable focus $\mathcal{O}_{43}^{s}$, the saddle $\mathcal{O}_{43}^{u}$ and its unstable manifold $\left.\mathcal{W}_{ \pm}^{u}\left(\mathcal{O}_{43}^{u}\right)\right)$. The basins $\mathcal{B}\left(\mathcal{C}_{133}\right)$ and $\mathcal{B}\left(\mathcal{C}_{43}\right)$ are separated from each other by a repelling closed invariant curve $\mathcal{C}_{\infty}^{2}$. The structure of the phase space close to this curve is similar to the one close to $\mathcal{C}_{\infty}^{1}$.

To sum up, at the considered parameter values the map has five attracting closed invariant curves, namely $\mathcal{C}_{4}, \mathcal{C}_{43}, \mathcal{C}_{47}, \mathcal{C}_{90}, \mathcal{C}_{133}$, whose basins are separated from each other by four repelling invariant sets, namely, two repelling closed invariant curves $\mathcal{C}_{\infty}^{1}$ and $\mathcal{C}_{\infty}^{2}$, and two chaotic saddles $\mathcal{S}_{\text {ch }}^{1}$ and $\mathcal{S}_{\text {ch }}^{2}$.

It is worth mentioning that the presented relationship of the appearance of the nested invariant curves to the period adding explains also the order in which the invariant curves are nested into each other. Indeed, typically, the size of the cycles in a period adding structure changes monotonously under parameter variation (for example, in Fig. 2, the size of the cycles is monotonously decreasing with increasing parameter values). Accordingly, it can be expected that at the parameter point where the cycles coexist, they are located in the state space in the same order as they appear in the period adding structure. For example, the size of the invariant curve $\mathcal{C}_{90}$ at the parameter values corresponding to Fig. 2 is larger than the size of the invariant curve $\mathcal{C}_{47}$ but smaller than the size of $\mathcal{C}_{43}$. At the point $q$ where all these invariant curves coexist, the invariant curve $\mathcal{C}_{90}$ is located between invariant curves $\mathcal{C}_{43}$ and $\mathcal{C}_{47}$. The same applies to the other invariant curves existing at this parameter value as well.

\section{Summary}

Multistability, i.e. coexistence of attractors, is a typical phenomenon in nonlinear dynamics. Recently, Zhusubaliyev et al. Zhusubalivev et al., 2011; Zhusubaliyev \& Mosekilde, 2015] have found that piecewise smooth dynamic systems can exhibit an unusual type of multistability. It has been reported that coexisting stable closed invariant curves embedded one into the other can arise via several global and border-collision bifurcations.

In the present work we examined a possible mechanism leading to the appearance of several attracting closed invariant curves nested into each other in the piecewise-linear 2D normal form (1). We have demonstrated that this kind of multistability may be related to the well-known period adding bifurcation structure. The nested attracting closed invariant curves appear as a result of a combination of the following effects: on one hand, the period adding structure is already deformed so that the periodicity regions forming this structure overlap, and on the other hand, the closed invariant curves existing in some of these periodicity regions are still not destroyed.

It is worth noting how the discussed mechanism leading to the appearance of nested invariant curves differs from the situation presented in [Zhusubaliyev et al., 2011; Zhusubaliyev \& Mosekilde, 2015]. In the cited work, the multistability is caused by the presence of two period adding structures. In that case, the regions belonging to the same structure, do not overlap, so that the coexisting cycles belong to different period adding structures. Accordingly, not more than two coexisting attracting nested invariant curves have been reported. By contrast, in the case that the cycles forming the invariant curves belong to the same period adding structure, the number of curves can be much higher.

Moreover, we have demonstrated that the basins of attraction of the nested attracting invariant curves may be separated from each other not only by repelling closed invariant curves, as previously reported. The boundary between basins may also be formed by a chaotic saddle from which also the basins of further attracting cycles issue. Both mechanisms appear to be quite generic; in the presented example each of them appear twice.

As the parameter combination considered in this paper satisfies conditions (2), for $\mu<0$ the map has an attracting fixed point. Therefore, a variation of the parameter $\mu$ from negative to positive values leads in the presented example to a special kind of border collision bifurcations, namely a multiple attractor-bifurcation in which several attracting nested closed invariant curves are simultaneously created from a stable fixed point. 


\section{Acknowledgment}

The work of V. Avrutin was supported by the German Research Foundation within the scope of the project "Generic bifurcation structures in piecewise-smooth maps with extremely high number of borders in theory and applications for power converter systems".

\section{References}

Agliari, A., Bischi, G.-I., Dieci, R. \& Gardini, L. [2005] "Global bifurcations of closed invariant curves in twodimensional maps: A computer assisted study," Int. J. Bifurcation and Chaos 15, 1285-1328.

Avrutin, V., Schanz, M. \& Banerjee, S. [2011] "Occurrence of multiple attractor bifurcations in the twodimensional piecewise linear normal form map," Nonlin. Dyn. 67, 293-307.

Banerjee, S. \& Verghese, G. C. [2001] Nonlinear Phenomena in Power Electronics - Attractors, Bifurcations, Chaos, and Nonlinear Control (IEEE Press).

Blażejczyk-Okolewska, B. \& Kapitaniak, T. [1998] "Coexisting attractors of impact oscillator," Chaos Solit. Fract. 9, 1439-1443.

Brambilla, M., Lugiato, L. A., Penna, V., Prati, F., Tamm, C. \& Weiss, C. O. [1991] "Transverse laser patterns. II. Variational principle for pattern selection, spatial multistability, and laser hydrodynamics," Phys. Rev. A 43, 5114-5120.

Campbell, D., Galeeva, R., Tresser, C. \& Uherka, D. [1996] "Piecewise linear models for the quasiperiodic transition to chaos," Chaos 6, 121-154.

Do, Y. \& Lai, Y.-C. [2008] "Multistability and arithmetically period-adding bifurcations in piecewise smooth dynamical systems," Chaos 18, 043107.

Dutta, M., Nusse, H. E., Ott, E., Yorke, J. A. \& Yuan, G. [1999] "Multiple attractor bifurcations: A source of unpredictability in piecewise smooth systems," Phys. Rev. Lett. 83, 4281-4284.

Feudel, U., Grebogi, C., Hunt, B. R. \& Yorke, J. A. [1996] "Map with more than 100 coexisting low-period periodic attractors," Phys. Rev. E 54, 71-81.

Feudel, U. [2008] "Complex dynamics in multistable systems," Int. J. Bifurcation and Chaos 18, 16071626.

Feudel, U., Pisarchik, A. \& Showalter, K. [2018] "Multistability and tipping: From mathematics and physics to climate and brain - Minireview and preface to the focus issue," Chaos 28, 033501.

Foss, J., Longtin, A., Mensour, B. \& Milton, J. [1996] "Multistability and delayed recurrent loops," Phys. Rev. Lett. 76, 708-711.
Hens, C., Dana, S. \& Feudel, U. [2015] "Extreme multistability: Attractor manipulation and robustness," Chaos 25, 053112.

Kapitaniak, T. \& Maistrenko, Y. [1998] "Multiple choice bifurcations as a source of unpredictability in dynamical systems," Phys. Rev. E 58, 5161-5163.

Kuznetsov, Y. [2004] Elements of Applied Bifurcation Theory, 3rd edition (Springer).

Liu, Y. \& Chávez, J. P. [2017] "Controlling coexisting attractors of an impacting system via linear augmentation," Physica D 348, 1-11.

Marmillot, P., Kaufman, M. \& Hervagault, J.-F. [1991] "Multiple steady states and dissipative structures in a circular and linear array of three cells: Numerical and experimental approaches," J. Chem. Phys. 95, $1206-1214$.

Nusse, H. E. \& Yorke, J. A. [1992] "Border-collision bifurcations including 'period two to period three' bifurcation for piecewise smooth systems," Physica D 57, 39-57.

Pavlovskaia, E., Ing, J., Wiercigroch, M. \& Banerjee, S. [2010] "Complex dynamics of bilinear oscillator close to grazing," Int. J. Bifurcation and Chaos 20, 38013817.

Pisarchik, A. \& Feudel, U. [2014] "Control of multistability," Phys. Rep. 540, 167-218.

Prengel, F., Wacker, A. \& Schöll, E. [1994] "Simple model for multistability and domain formation in semiconductor superlattices," Phys. Rev. B 50, 17051712 .

Simpson, D. \& Meiss, J. [2009] "Shrinking point bifurcations of resonance tongues for piecewise-smooth, continuous maps," Nonlinearity 22, 1123-1144.

Simpson, D. J. W. [2014] "Sequences of periodic solutions and infinitely many coexisting attractors in the border-collision normal form," Int. J. Bifurcation and Chaos 24, 1430018-1-18.

Simpson, D. [2017] "The structure of mode-locking regions of piecewise-linear continuous maps: I. Nearby mode-locking regions and shrinking points," Nonlinearity 30, 382-444.

Simpson, D. [2018] "The structure of mode-locking regions of piecewise-linear continuous maps: II. Skew sawtooth maps," Nonlinearity 31, 1905-1939.

Yang, W.-N. \& Hao, B.-L. [1987] "How the Arnol'd tongues become sausages in a piecewise linear circle map," Commun. Theor. Phys. 8, 1-15.

Zhusubaliyev, Zh. T., Soukhoterin, E. \& Mosekilde, E. [2002] "Border-collision bifurcations on a twodimensional torus," Chaos Solit. Fract. 13, 18891915.

Zhusubaliyev, Zh. T. \& Mosekilde, E. [2003] Bifurcations and Chaos in Piecewise-Smooth Dynamical Systems, World Scientific Series on Nonlinear Science A, Vol. 44 (World Scientific). 
Zhusubaliyev, Zh. T., Mosekilde, E., Maitya, S., Mohanan, S. \& Banerjee, S. [2006] "Border collision route to quasiperiodicity: Numerical investigation and experimental confirmation," Chaos 16, 023122.

Zhusubaliyev, Zh. T. \& Mosekilde, E. [2008] "Equilibrium-torus bifurcation in nonsmooth system," Physica D 237, 930-936.
Zhusubaliyev, Zh. T., Mosekilde, E. \& Yahochkina, O. [2011] "Torus bifurcations in multilevel converter systems," Int. J. Bifurcation and Chaos 21, 2343-2356.

Zhusubaliyev, Zh. T. \& Mosekilde, E. [2015] "Multistability and hidden attractors in a multilevel DC/DC converter," Math. Comput. Simul. 109, 32-45. 